ARTICLE

DOI: $10.1038 / s 41467-017-01704-z$

\title{
AIP limits neurotransmitter release by inhibiting calcium bursts from the ryanodine receptor
}

\author{
Bojun Chen ${ }^{1}$, Ping Liu ${ }^{1}$, Edward J. Hujber ${ }^{2,3}$, Yan Li ${ }^{1}$, Erik M. Jorgensen ${ }^{2,3}$ \& Zhao-Wen Wang ${ }^{1}$
}

Pituitary tumors are frequently associated with mutations in the AIP gene and are sometimes associated with hypersecretion of growth hormone. It is unclear whether other factors besides an enlarged pituitary contribute to the hypersecretion. In a genetic screen for suppressors of reduced neurotransmitter release, we identified a mutation in Caenorhabditis elegans AIPR-1 (AIP-related-1), which causes profound increases in evoked and spontaneous neurotransmitter release, a high frequency of spontaneous calcium transients in motor neurons and an enlarged readily releasable pool of vesicles. Calcium bursts and hypersecretion are reversed by mutations in the ryanodine receptor but not in the voltage-gated calcium channel, indicating that these phenotypes are caused by a leaky ryanodine receptor. AIPR-1 is physically associated with the ryanodine receptor at synapses. Finally, the phenotypes in aipr-1 mutants can be rescued by presynaptic expression of mouse AIP, demonstrating that a conserved function of AIP proteins is to inhibit calcium release from ryanodine receptors.

\footnotetext{
${ }^{1}$ Department of Neuroscience, University of Connecticut Health Center, Farmington, CT 06030, USA. ${ }^{2}$ Department of Biology, University of Utah, Salt Lake City, UT 84112, USA. ${ }^{3}$ Howard Hughes Medical Institute, University of Utah, Salt Lake City, UT 84112, USA. Correspondence and requests for materials should be addressed to Z.-W.W. (email: zwwang@uchc.edu)
} 
C alcium has pivotal roles in triggering neurotransmitter release. Calcium influx through voltage-gated calcium channels is the main source of calcium driving synaptic vesicle fusion. In addition, smooth endoplasmic reticulum (ER) extends into axon terminals and ryanodine receptor-mediated calcium release from these internal stores regulates synaptic activity $^{1-4}$. Presynaptic ryanodine receptors release calcium in a calcium-dependent manner and can shape synaptic transmission $^{5}$. Specifically, they may enhance spontaneous and evoked neurotransmitter release, promote synchrony of vesicle fusion, and contribute to short-term synaptic plasticity ${ }^{5-8}$. Increased calcium release from ryanodine receptors is considered to be a central factor in the pathogenesis of Alzheimer's disease ${ }^{9,10}$. Thus, identifying molecules that inhibit the ryanodine receptor is important for understanding the mechanisms of both physiological control of synaptic function and diseases associated with leaky RyRs.

The ryanodine receptor is a tetramer of ryanodine receptor proteins (RyR). Human and mouse each have three genes encoding different isoforms of ryanodine receptor proteins: RyR1, RyR2, and RyR3, which display both tissue-specific and overlapping expression patterns. RyR1 and RyR2 are expressed in skeletal and cardiac muscles, respectively, and all three isoforms are expressed in the brain ${ }^{11}$. The physiological functions of ryanodine receptors depend on their interactions with a variety of other proteins. For example, calcium release from ryanodine receptors is modulated by their association with calstabin 1 (FKBP12) and calstabin 2 (FKBP12.6) ${ }^{11,12}$, which are immunophilins containing a prolyl isomerase domain with enzyme activity $^{13}$. Dissociation of calstabins from the ryanodine receptor causes increased open probability of the channel ${ }^{14,15}$. In mice with calstabin 2 mutations, leaky ryanodine receptors may cause cognitive dysfunction ${ }^{15,16}$ and fatal cardiac arrhythmia ${ }^{14}$. Treatment with a drug that enhances the binding of calstabin 2 to ryanodine receptors can prevent or attenuate seizures, cardiac arrhythmias, and sudden cardiac death caused by mutations of RyR $2^{17}$. The inhibition of ryanodine receptors by calstabins appears to be a conserved mechanism, because ryanodine receptors are similarly regulated by a homolog of calstabins (FKB2) in C. elegans ${ }^{18}$.

AIP (aryl hydrocarbon receptor-interacting protein) belongs to the TPR (tetratricopeptide repeat) family of co-chaperones (Fig. 1a). It contains a prolyl-isomerase-like domain on the $\mathrm{N}$ terminal, and a TPR domain and a helical extension to the TPR domain called the " $\alpha-7$ helix" on the C-terminal ${ }^{19}$ (Fig. 1c). Although the prolyl isomerase domain of AIP resembles those of calstabins, it does not have the enzyme activity ${ }^{20}$. The TPR domain contains three TPR motifs ${ }^{20}$, which binds the C-terminal residues of the chaperone HSP90 21 . AIP is a suppressor of pituitary tumors ${ }^{22}$. Affected individuals are heterozygous for the mutant allele; the tumors are usually homozygous due to loss of heterozygosity in the pituitary gland in both humans and mice ${ }^{22-}$
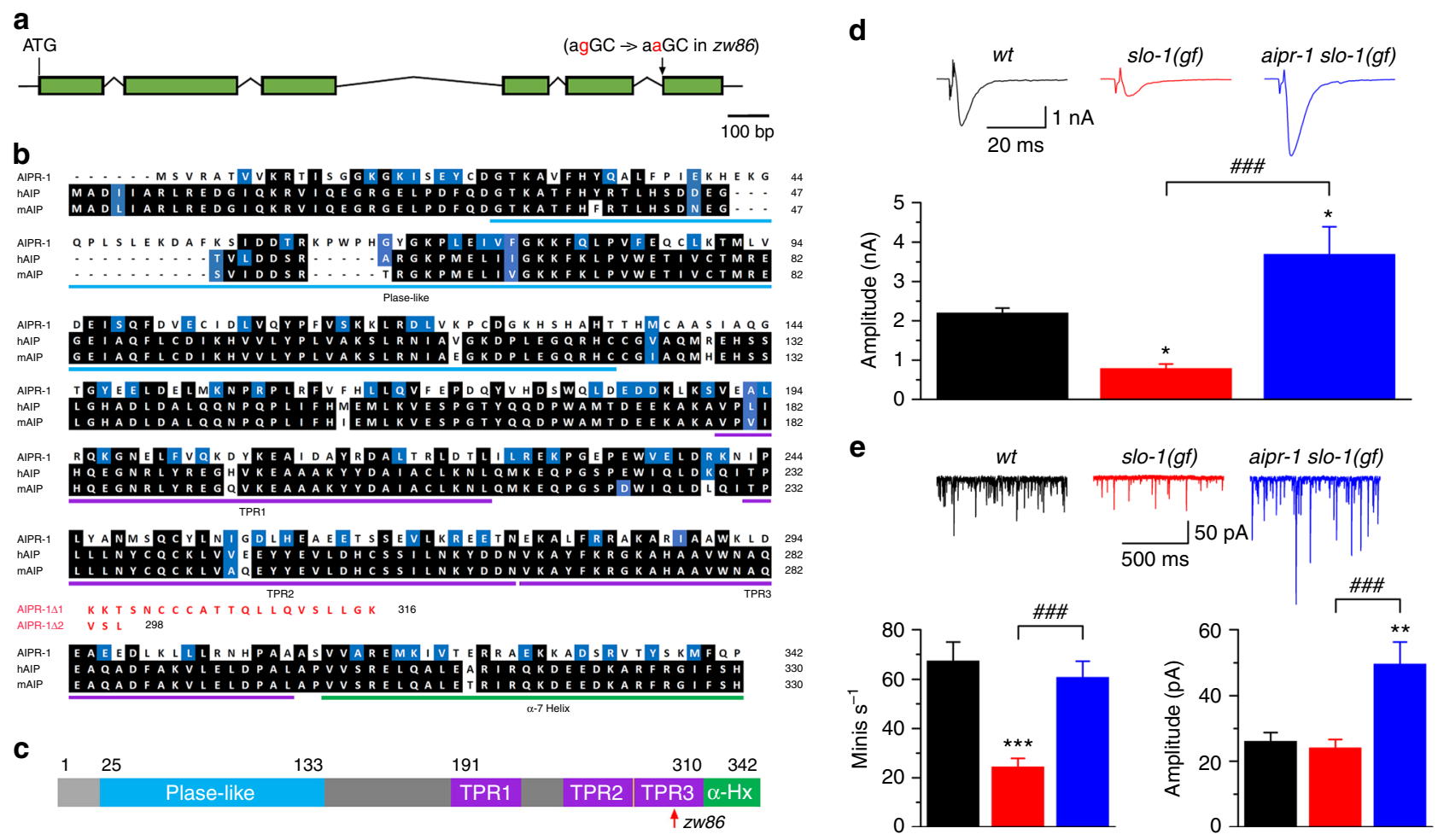

Fig. 1 The effects of aipr-1(zw86) mutation on synaptic phenotypes of slo-1 gain-of-function ( $g$ f). a Schematic diagram showing the exon and intron organization of aipr-1 (GenBank: NP_495339.1). zw86 is a G to A transition in the splice acceptor site before the last exon. b Alignment of amino acid sequences between AIPR-1, human AIP (hAIP) (GenBank: ACN38897.1), and mouse AIP (mAIP) (GenBank: AAH75614.1). AIPR-1 is 35\% identical to hAIP. Identical residues are highlighted in black, whereas similar ones (in size, acidity, or polarity) in blue. The prolyl cis-trans isomerase-like domain (Plase-like), tetratricopeptide repeat (TPR) motifs, and the C-terminal $\alpha-7$ helix $(\alpha-\mathrm{Hx})$ of the TPR repeat structure are underlined. aipr-1(zw86) does not make wildtype AIPR-1 but may produce two alternative isoforms truncated after glutamate (E) 295 (AIPR-1 11 and AIPR-1 $\Delta 2$ ) with additional out-of-frame amino acid residues. c Diagram of AIPR-1 domain structure. The red arrow indicates the location of AIPR-1 truncation in zw86. $\mathbf{d}$ Comparison of evoked current amplitude among wild-type (wt), slo-1(gf), and the slo-1(gf) aipr-1(zw86) double mutant. $n=8$ in all groups. e Comparison of the frequency and mean amplitude of spontaneous minis among the three groups. $n=8$ in all groups. Data are shown as mean \pm SEM. ${ }^{\star} p<0.05,{ }^{\star \star} p<0.01,{ }^{\star \star \star} p<0.001$ compared with wt; \#\#\# $p<0.001$ compared between with slo-1(gf) (one-way ANOVA followed by Tukey's post-hoc test) 
24. The aryl hydrocarbon receptor-cAMP-phosphodiesterase pathway appears to be important for why AIP mutations cause pituitary tumors ${ }^{25}$. Patients with pituitary tumors including those caused by mutations of the AIP gene frequently exhibit acromegaly and gigantism due to hypersecretion of growth hormone $22,26,27$. It is unknown whether any other factors besides the increased pituitary size have a role in the hypersecretion of growth hormone. Although at least 20 interacting proteins have been identified for AIP, none suggest an obvious direct mechanism for growth hormone hypersecretion ${ }^{20}$. In addition, AIP is abundantly expressed in the human brain (http://human. brain-map.org/) but little is known about its physiological roles in the nervous system.

Here we demonstrate that AIPR-1 (AIP-related 1), the worm ortholog of human AIP, is an inhibitor of presynaptic ryanodine receptors. We find that a mutation in AIPR-1 results in increased release of neurotransmitter and this effect is occluded by a lossof-function mutation of the ryanodine receptor but not of a presynaptic voltage-gated calcium channel. In addition, the AIPR-1 mutation causes increases in synaptic vesicle number and the readily releasable pool size through a ryanodine receptorindependent pathway. This study identifies AIPR-1/AIP as a critical regulator of neurotransmitter release.

\section{Results}

AIPR-1 is a potent inhibitor of synaptic transmission. We identified aipr-1 in a forward genetic screen for mutants that increased neurotransmitter release in a sensitized background. In a worm strain expressing a hyperactive BK channel SLO-1, a calcium-activated potassium channel that hyperpolarizes the synaptic terminal and limits exocytosis, neurotransmitter release is greatly reduced, causing a lethargic phenotype ${ }^{28}$. We screened for suppressors of the sluggish phenotype and identified the mutation $z w 86$, which ameliorated the locomotion defect of the slo-1(gf) strain. Mapping, sequencing, and rescue experiments established that the $z w 86$ mutation is an allele of the gene aipr-1, the worm ortholog of mammalian AIP (Fig. 1a-c). $z w 86$ is a $\mathrm{G}$ to A transition in the splice acceptor site before the last exon (Fig. 1a). Sequencing of aipr-1(zw86) complementary DNA revealed that the mutant does not make wild-type AIPR-1 but may produce two alternative isoforms with frame shifts (Fig. 1b). aipr-1 is the third gene in an operon of four genes, including epg5 (C56C10.12 + C56C10.11), dnj-8, aipr-1, and C56C10.9. The $z w 86$ mutation did not affect mRNA levels of the other three genes in the operon (Supplementary Fig. 1). The aipr-1 mutant is hyperactive; quantification with an automated worm tracker ${ }^{29}$ showed that locomotion speed of the mutant doubled compared to the wild type (Supplementary Fig. 2). a

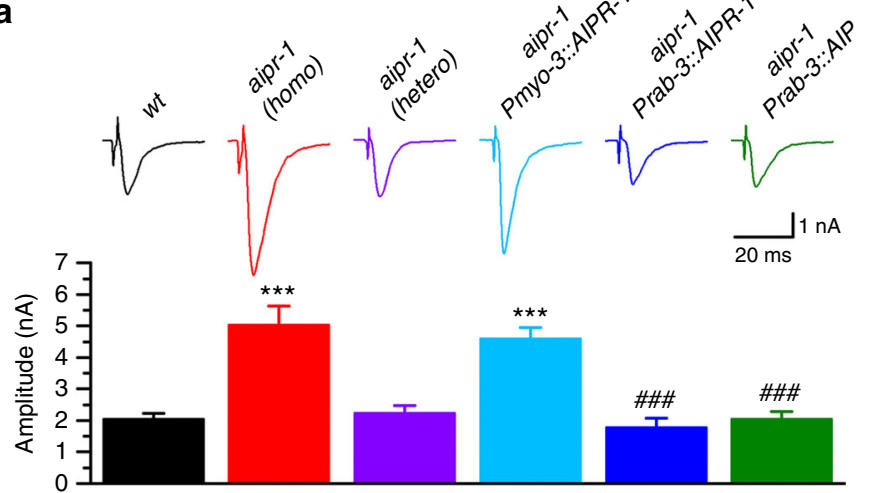

b
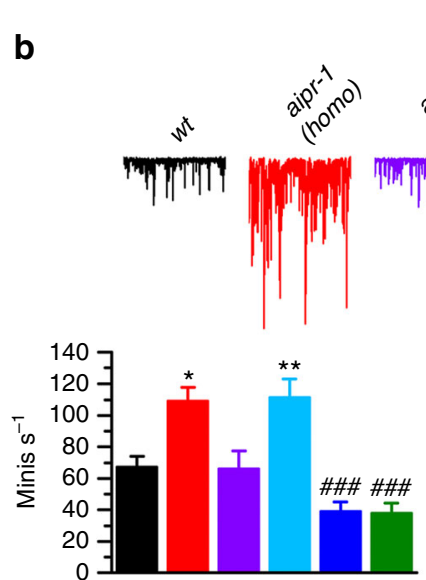
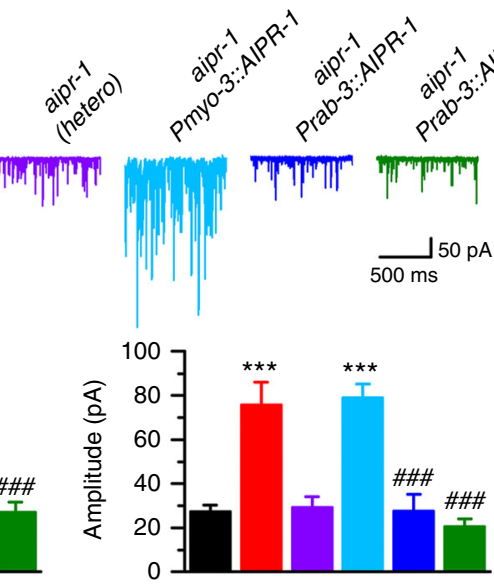

C

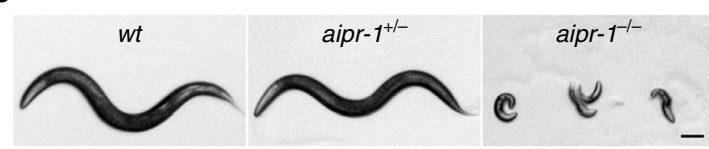

d
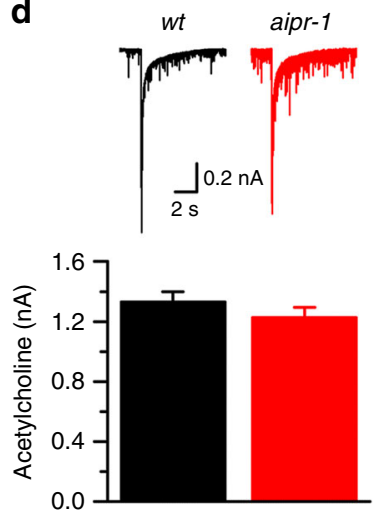

e

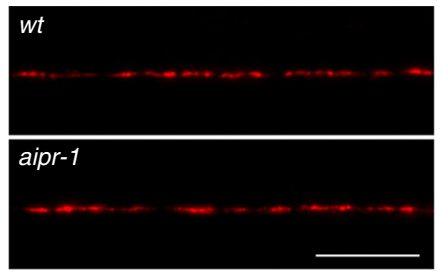

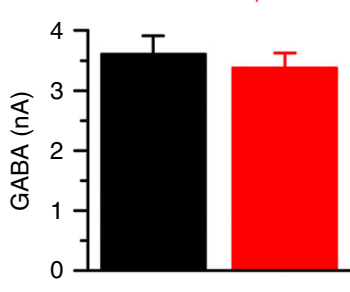
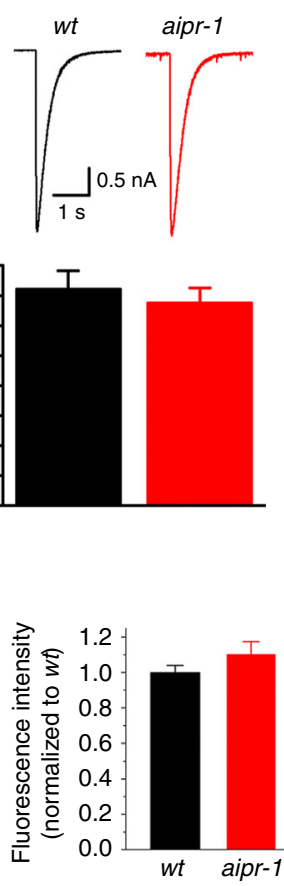

Fig. 2 Augmented synaptic transmission in aipr-1(zw86). a, b Comparison of evoked currents a and spontaneous miniature currents (minis) $\mathbf{b}$ at the neuromuscular junction among wild type $(w t)(n=7)$, homozygous aipr-1(zw86) $(n=7)$, heterozygous aipr-1(zw86) $(n=7)$, and aipr-1(zw86) rescued either in neurons (Prab-3::AIRP-1, $n=7$; Prab-3::AIP, $n=9$ ) or body-wall muscle cells (Pmyo-3::AIPR-1, $n=7$ ). c Homozygous aipr-1 knockout (aipr-1\%/) worms arrest at early larval stages. The knockout results from a 2 bp deletion in the first exon of aipr-1. Scale bar, $100 \mu \mathrm{m}$. $\mathbf{d}$ Comparison of muscle inward current in response to exogenous acetylcholine $(100 \mu \mathrm{M})$ and GABA $(100 \mu \mathrm{M})$ between wt (ACh, $n=7 ; \mathrm{GABA}, n=7)$ and aipr-1(zw86) (ACh, $n=8 ; \mathrm{GABA}$, $n=8$ ). Data are shown as mean \pm SEM. ${ }^{*} p<0.05,{ }^{* \star} p<0.01,{ }^{\star \star *} p<0.001$ compared with wt; \#\#\#p<0.001 compared with aipr-1(zw86) (one-way ANOVA followed by Tukey's post-hoc test). e tagRFP-labeled muscle acetylcholine receptor (UNC-29) distribution and fluorescence intensity are normal in aipr-1(zw86) (wt, $n=20$; aipr-1, $n=23$, unpaired $t$-test). Scale bar, $10 \mu \mathrm{m}$ 

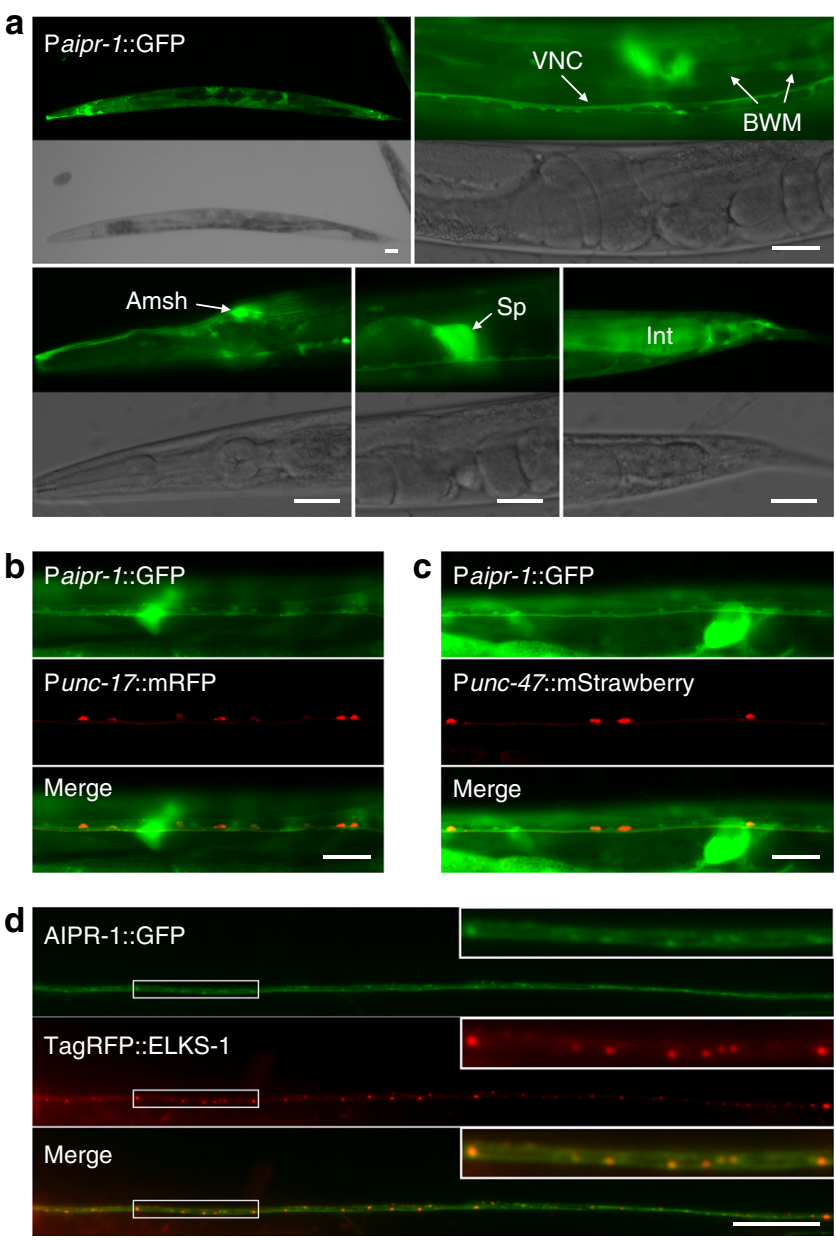

Fig. 3 Expression and subcellular localization of AIPR-1. a A GFP reporter, expressed under the control of genomic DNA upstream of the aipr-1 initiation site, showed expression in many cell types, such as motor neurons along the ventral nerve cord (VNC), body-wall muscle cells (BWM), amphid sheath cells (Amsh), spermatheca (Sp), and the intestine (Int). b, c AIPR-1 is expressed in all acetylcholine and GABA motor neurons in the ventral nerve cord. Acetylcholine and GABA motor neurons were labeled by expressing mRFP and mStrawberry under the control of Punc-17 and Punc47, respectively. Images were taken from a segment anterior $\mathbf{b}$ and posterior $\mathbf{c}$ to the vulva. d, AIPR-1::GFP displayed both diffuse and punctate distribution along the dorsal nerve cord, and the GFP puncta colocalized with the presynaptic marker TagRFP::ELKS-1. Images were taken from a dorsal segment anterior to the vulva. Scale bars, $20 \mu \mathrm{m}$ a-c and $10 \mu \mathrm{m} \mathbf{d}$, e

The increased activity observed in the slo-1(gf) aipr-1(zw86) double mutant suggests that a potential physiological function of AIPR-1 is to limit synaptic activity. Indeed, electrophysiological recordings from the neuromuscular junction demonstrated that the $z w 86$ mutation increases synaptic transmission in slo- $1(g f)$ mutants. The frequency and mean amplitude of spontaneous miniature postsynaptic currents ('minis') and the amplitude of evoked postsynaptic currents are increased in the aipr-1 slo-1(gf) double mutant compared with slo-1(gf) single mutants (Fig. 1d, e).

The single mutant aipr-1(zw86), when outcrossed from slo-1 $(g f)$, exhibits a doubled evoked response ( $w t 2.1 \pm 0.2 \mathrm{nA}$; aipr-1 $4.9 \pm 0.5 \mathrm{nA})$, an increased frequency of spontaneous minis $(w t$ $66.9 \pm 11.3 \mathrm{~s} \mathrm{~s}^{-1}$; aipr-1 107.2 $\left.\pm 7.4 \mathrm{~s}^{-1}\right)$, and an increased amplitude of minis (wt $27.6 \pm 3.3 \mathrm{pA}$; aipr-1 $74.4 \pm 8.7 \mathrm{pA}$ ) compared with the wild type in the presence of $5 \mathrm{mM}\left[\mathrm{Ca}^{2+}\right]_{\mathrm{o}}$ (Fig. 2a, b). Both evoked responses and minis were normal in heterozygous aipr-1(zw86) (Fig. 2a, b), suggesting that the mutation is fully recessive. Despite being isolated as a suppressor of a hyperactive SLO-1 potassium channel, AIPR-1 does not act in the same pathway as SLO-1. If AIPR-1 were to inhibit neurotransmission by activating SLO-1, then aipr-1 mutants should have resembled slo-1 loss-of-function ( $l f)$ mutants but should not exhibit a more severe phenotype. However, synaptic phenotypes of aipr-1(zw86) are much more severe than those of slo-1 $(l f)$. Although the mutation of aipr-1 causes large increases in evoked responses and in the frequency and mean amplitude of minis at both 0.5 and 5.0 $\mathrm{mM}\left[\mathrm{Ca}^{2+}\right]_{\mathrm{o}}$, slo-1(lf) shows only smaller increases in evoked responses and the frequency of minis at the lower $\left[\mathrm{Ca}^{2+}\right]_{\mathrm{o}}$ (Supplementary Fig. 3a, b). Moreover, aipr-1(zw86) does not alter either SLO-1 expression or subcellular localization (Supplementary Fig. 3c-e), unlike other mutants that suppress SLO-1(gf) $28,30,31$. Thus, AIPR-1 inhibits neurotransmission through a different pathway than through the SLO-1 potassium channel.

Worm and mammalian AIP act presynaptically. Similar to the human protein, AIPR-1 is composed of an FKBP-type prolyl isomerase domain, a TPR domain with three TPR motifs and the associated " $\alpha-7$ " helix (Fig. 1c). cDNA sequencing indicates that the two incorrectly spliced isoforms with frame shifts disrupt the last TPR motif and the $\alpha-7$ helix (Fig. 1b). Similarly, most mutations of AIP in human patients disrupt or delete the last TPR motif and the $\alpha-7$ helix ${ }^{20,24}$. We generated a deletion allele of aipr-1 using CRISPR/Cas9; zw90 homozygous animals arrest at early larval stages (Fig. 2c), which suggest that aipr-1(zw86) is a hypomorph. Similarly, knockouts of the AIP gene in mouse are embryonic lethal ${ }^{32}$.

To test whether mammalian AIP can substitute for AIPR-1 function, we expressed mouse AIP in aipr-1(zw86) neurons under the control of the rab-3 promoter (Prab-3). Mouse AIP rescued the synaptic phenotypes (Fig. 2a, b) (evoked: aipr-1 $4.9 \pm 0.5 \mathrm{nA}$, aipr-1 Prab-3::AIPR-1 1.8 $\pm 0.3 \mathrm{pA}$, aipr-1 Prab-3:AIP $2.0 \pm 0.2$ pA; minis: aipr-1 $74.4 \pm 8.7 \mathrm{pA}$, aipr-1 Prab-3:AIPR-1 $27.7 \pm 7.5$ pA, aipr-1 Prab-3:AIP $20.7 \pm 3.2 \mathrm{pA}$; mini frequency: aipr-1 107.2 $\pm 7.4 \mathrm{~s}^{-1}$, aipr-1 Prab-3:AIPR-1 $39.5 \pm 5.5 \mathrm{~s}^{-1}$, aipr-1 Prab3:AIP $\left.38.3 \pm 6.0 \mathrm{~s}^{-1}\right)$. The rescue of aipr-1(zw86) synaptic phenotypes by mouse AIP suggests that the proteins have similar roles in worms and mammals. Furthermore, rescue in neurons suggests that AIPR-1 acts presynaptically rather than in the postsynaptic muscles. Consistent with this conclusion, expression of AIPR-1 in muscles under the control of the myo-3 promoter (Pmyo-3) did not rescue aipr-1 mutants (evoked: $4.6 \pm 0.3 \mathrm{pA}$; minis: $79.3 \pm 5.9 \mathrm{pA}$; mini frequency $111.8 \pm 11.2 \mathrm{~s}^{-1}$ (Fig. 2a, b). Furthermore, direct application of either acetylcholine or $\gamma$ aminobutyric acid (GABA) onto muscle produces normal currents in aipr-1(zw86) (Fig. 2d), and the expression of a tagRFP-tagged UNC-2933, which is a key subunit of muscle acetylcholine receptor ${ }^{34}$, was similar between the wild type and aipr-1(zw86) (Fig. 2e), suggesting that muscle physiology is normal.

The aipr-1(zw86) mutants do not exhibit morphological changes in acetylcholine and GABA motor neurons (Supplementary Fig. 4a, b) or an increase in the density of presynaptic sites (Supplementary Fig. 4c, d), suggesting that hypersecretion is caused by a defect in synaptic function rather than development. Because both acetylcholine and GABA neurons synapse onto muscles, we recorded acetylcholine and GABA minis separately. Minis from both neurotransmitters exhibit higher frequencies and larger amplitudes in aipr-1(zw86) than in the wild type (Supplementary Fig. 5), indicating that AIPR-1 is not restricted to a particular synaptic type. 
a

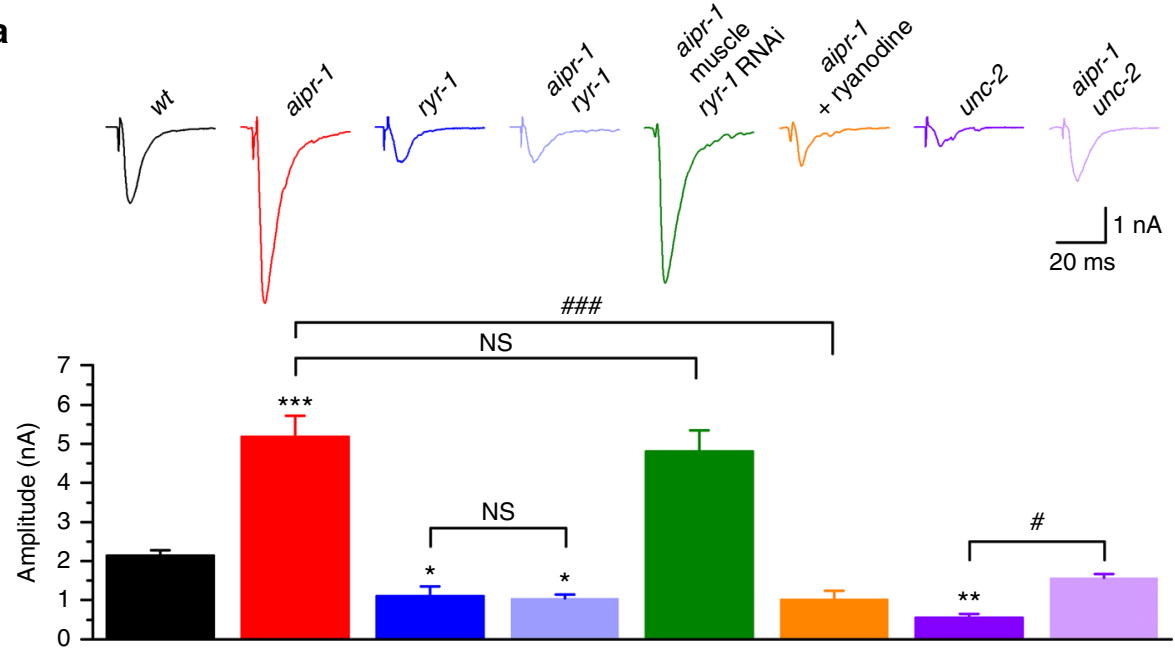

b
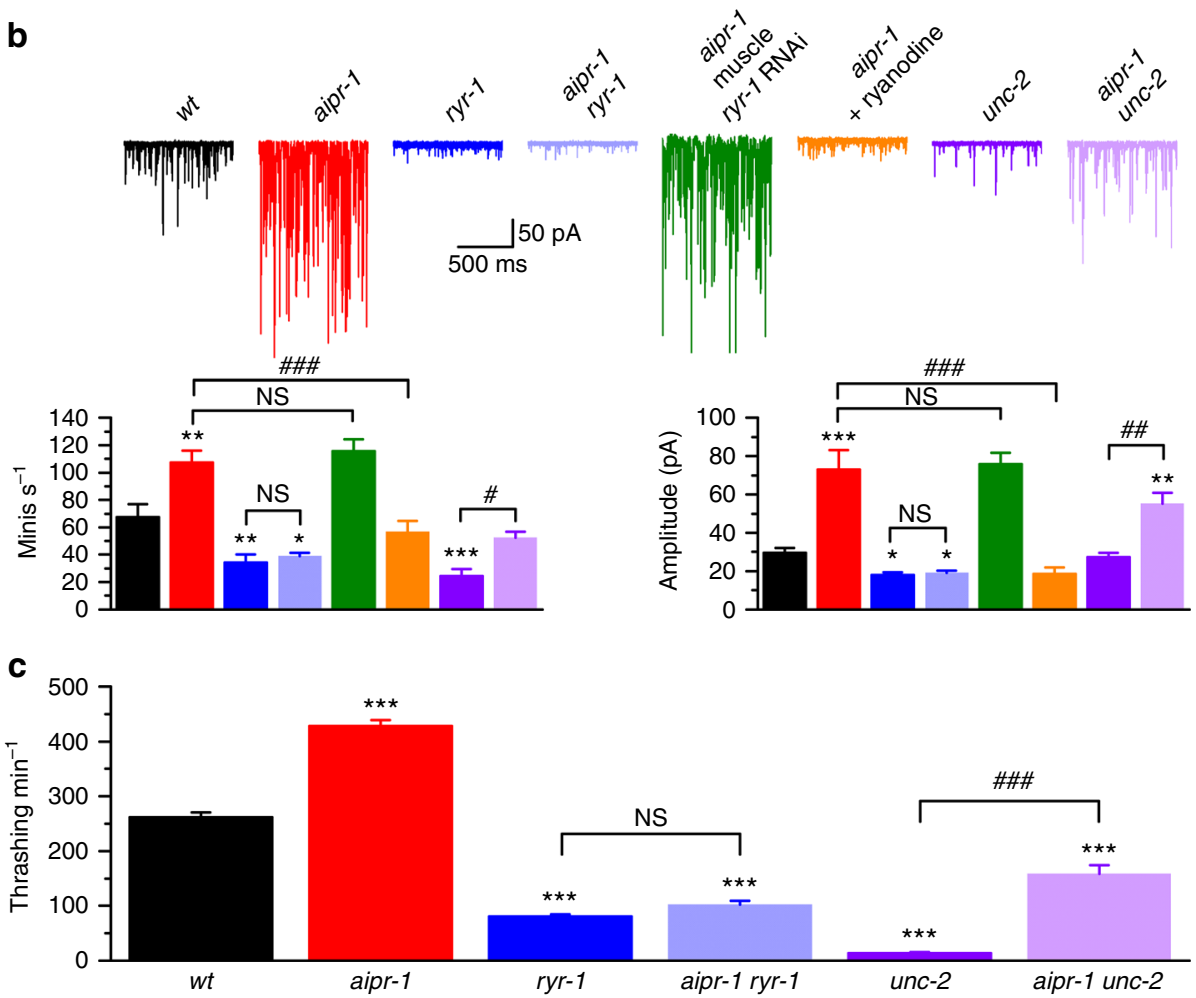

Fig. 4 Mutation of RYR-1 but not UNC-2 occluded synaptic and behavioral phenotypes of aipr-1(zw86). $\mathbf{a}, \mathbf{b}$ Comparison of evoked currents a and spontaneous miniature currents (minis) b among wild type $(w t)(n=8)$, aipr-1(86) $(n=7)$, ryr-1(e540) $(n=7)$, aipr-1(zw86) ryr-1(e540) ( $n=7)$, aipr-1 (zw86) with muscle ryr-1 RNAi $(n=7)$, aipr-1(zw86) treated with ryanodine $(100 \mu \mathrm{M})(n=8)$, unc-2(e55) $(n=9)$, and aipr-1(zw86) unc-2(e55) ( $n=7)$. c Comparison of thrashing rates among these groups $\left(n=12\right.$ or 13). Data are shown as mean \pm SEM. ${ }^{*} p<0.05,{ }^{\star *} p<0.01$, ${ }^{\star \star *} p<0.001$ compared with wt; ${ }^{\#} p<0.05,{ }^{\# \#} p<0.01,{ }^{\# \#} p<0.001$ compared between indicated groups; NS $p>0.05$ (one-way ANOVA followed by Tukey's post-hoc test)

To determine the expression pattern of AIPR-1, we generated a transcriptional reporter in an extrachromosomal array by in vivo recombination. A fosmid (WRM065bd01) containing the entire operon was coinjected with a plasmid containing homologous DNA encompassing $0.5 \mathrm{~kb}$ upstream of the aipr-1 start codon fused to green fluorescent protein (GFP). In transgenic worms, GFP was observed in a variety of cell types, including neurons, body muscles, amphid sheath cells, spermatheca, and the intestine (Fig. 3a). Both acetylcholine and GABA motor neurons express aipr-1, as indicated by the co-labeling of these neurons by a red fluorescent protein expressed under the control of cell-specific promoters (Fig. 3b, c). To determine the subcellular localization of AIPR-1, we tagged the protein with GFP at either the N or C terminus using CRISPR/Cas9. Both strains exhibit wild-type behavior, suggesting that the fusion proteins are functional. Electrophysiological responses were tested in the AIPR-1::GFP strain; evoked responses and minis were normal (Supplementary Fig. 6a, b). However, only very dim GFP fluorescence could be detected in these strains (Supplementary Fig. 6c). The lack of a strong GFP signal in the nerve cords prevented us from analyzing synaptic localization of AIPR-1. To determine AIPR-1 subcellular localization in neurons, we overexpressed AIPR-1::GFP under the control of the panneuronal rab-3 promoter. The tagged protein 
was found throughout axons and was enriched at synapses, as determined by colocalization with a presynaptic marker (Fig. 3d).

AIPR-1 inhibits calcium bursts from ryanodine receptors. The electrophysiological data suggest that AIPR-1 normally inhibits synaptic transmission. What then is the molecular target of AIPR-1? The prolyl isomerase-like domains of AIP resemble the prolyl isomerase domains of calstabin 1 and calstabin $2^{20}$. Because calstabins inhibit ryanodine receptor-mediated $\mathrm{Ca}^{2+}$ release from the $\mathrm{ER}^{14,15,35,36}$, the absence of AIPR-1 might also disinhibit the ryanodine receptor, causing frequent calcium bursts and increased exocytosis. We therefore tested whether AIPR-1 acts via the sole ryanodine receptor of the worm (called UNC-68 or RYR-1, we will use RYR-1 henceforth for clarity). The ryr-1 gene generates transcripts from two promoters (www.wormbase. org). Reporter constructs demonstrated that the upstream promoter is expressed in muscle, whereas the downstream promoter is expressed in neurons (Supplementary Fig. 7). To determine whether the ER extends into synaptic regions, we coexpressed an ER marker (mStrawberry::PISY-1) and a presynaptic marker (GFP::ELKS-1) in motor neurons. We found that the ER marker was distributed throughout the dorsal nerve cord, including synaptic regions (Supplementary Fig. 8), which is consistent with a presynaptic role for RYR-1.

Loss of ryanodine receptor function in both mammals and worms cause synaptic phenotypes opposite to those in aipr-1 $(z w 86)^{6-8,37}$. Specifically in worms, we see decreases in the amplitude of evoked currents and in the frequency and amplitude of spontaneous minis in mutants of $r y r-1^{8}$ (Fig. $4 \mathrm{a}, \mathrm{b}$ ). The profound increases in evoked and spontaneous currents in aipr-1 mutants are completely abolished by ryr-1(e540), a putative ryr-1null mutant ${ }^{38}$, and the double mutant exhibits reduced evoked and spontaneous currents identical to the ryr-1 single mutant (evoked: aipr-1 $5.1 \pm 0.5 \mathrm{nA}$; ryr-1 $1.1 \pm 0.2 \mathrm{nA}$; aipr-1 ryr-1 $1.1 \pm$ $0.1 \mathrm{nA}$; minis: aipr-1 $73.3 \pm 9.9 \mathrm{pA}$; ryr-1 $18.2 \pm 1.1 \mathrm{pA}$; aipr-1 ryr-1 $19.2 \pm 0.9 \mathrm{pA}$; mini frequency: aipr-1 $107.6 \pm 8.6 \mathrm{~s}^{-1}$; ryr-1 $34.5 \pm 5.8 \mathrm{~s}^{-1}$; aipr-1 ryr-1 $38.9 \pm 2.4 \mathrm{~s}^{-1}$ ) (Fig. 4a, b). Similarly, acute inhibition of ryanodine receptors using $100 \mu \mathrm{M}$ ryanodine abolished the increased evoked responses and minis in aipr-1 mutant (Fig. 4a, b), suggesting that the suppression of aipr-1 synaptic phenotypes by ryr-1 mutation is not due to a developmental defect. Knockdown of $r y r-1$ specifically in muscle had no effect on either evoked responses or minis of aipr-1(zw86) (Fig. 4a, b), suggesting that loss of presynaptic ryr-1 occludes the synaptic phenotypes of the aipr-1 mutant. Another major source of calcium at synapses is the $\mathrm{N}$-type voltage-gated calcium channel $\left(\mathrm{Ca}_{\mathrm{V}} 2\right)$ encoded by $u n c-2^{39-42}$. Loss-of-function mutations in $u n c-2$ also cause reduced evoked and spontaneous currents. However, in contrast to the aipr-1 ryr-1 mutant, the aipr-1 unc-2 double mutant exhibits increased neurotransmission compared with $u n c-2$ alone (Fig. $4 \mathrm{a}, \mathrm{b}$ ), suggesting that at least part of the AIPR-1 function does not require the N-type calcium channel. Finally, ryr-1 also suppresses the behavioral phenotypes of aipr-1. In solution, aipr-1(zw86) worms show a much higher thrashing rate compared to the wild type and this behavioral phenotype is suppressed by ryr-1 but not unc-2 mutations (Fig. 4c and Supplementary Movies 1-6). The specificity of ryr-1(lf) in suppressing both the synaptic and behavioral phenotypes of aipr$1(z w 86)$ suggests that AIPR-1 is an inhibitor of the RYR-1 channels.

To determine whether AIPR-1 inhibits calcium bursts from ryanodine receptors, we examined calcium transients in motor neurons using the $\mathrm{Ca}^{2+}$ indicator GCaMP $6 \mathrm{f}^{43}$. To knock down AIPR-1 expression, we performed RNA interference (RNAi). RNAi is expected to specifically act on aipr-1 transcripts although we cannot exclude the possibility that RNAi might affect other genes of the operon. To avoid potential complications from increased activity of upstream neurons, we performed motor neuron-specific RNAi for aipr-1, ryr-1, or both. The frequency of $\mathrm{Ca}^{2+}$ transients is more than doubled when aipr-1 is knocked down in A-type motor neurons and these frequent calcium bursts are occluded by $r y r-1$ knockdown (Fig. 5 and Supplementary Movies 7-10). These results suggest that reducing AIPR-1 function results in a "leaky" RYR-1, causing excessive $\mathrm{Ca}^{2+}$ release from the ER.
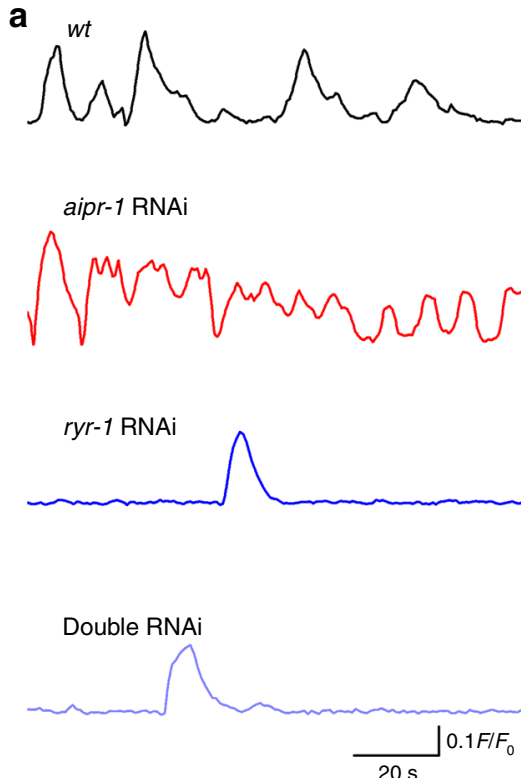
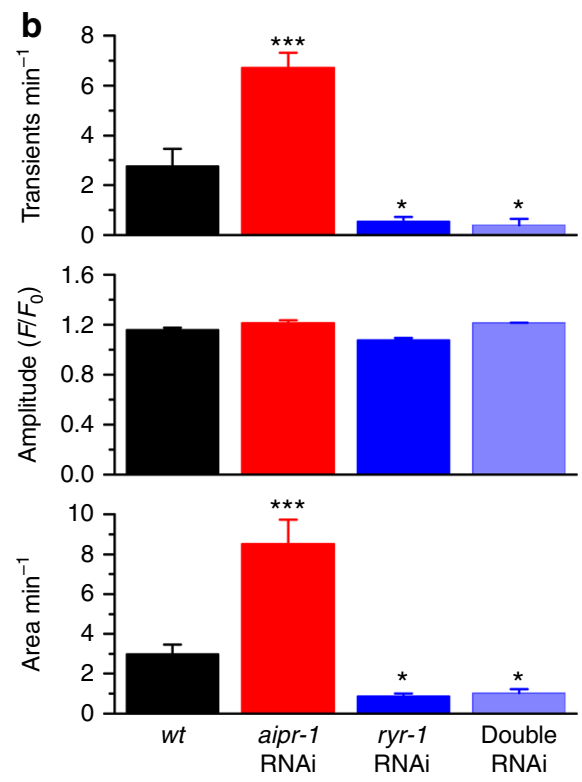

Fig. 5 AIPR-1 inhibits neuronal $\mathrm{Ca}^{2+}$ bursts from the ryanodine receptor. Expression of either aipr-1, ryr-1, or both genes was knocked down in A-type motor neurons using RNAi. a Representative traces of $\mathrm{Ca}^{2+}$ transients, determined in A-type motor neurons using GCaMP6f. b Bar graphs of calcium transient frequencies, amplitudes, and area in the wild type $(w t)(n=9)$, aipr-1 knockdown $(n=10)$, ryr-1 knockdown ( $n=6)$, and aipr-1 ryr-1 double knockdown ( $n=$ 6). Data are shown as mean \pm SEM. ${ }^{\star} p<0.05,{ }^{\star \star \star} p<0.001$ compared with wt; (one-way ANOVA followed by Tukey's post-hoc test) 

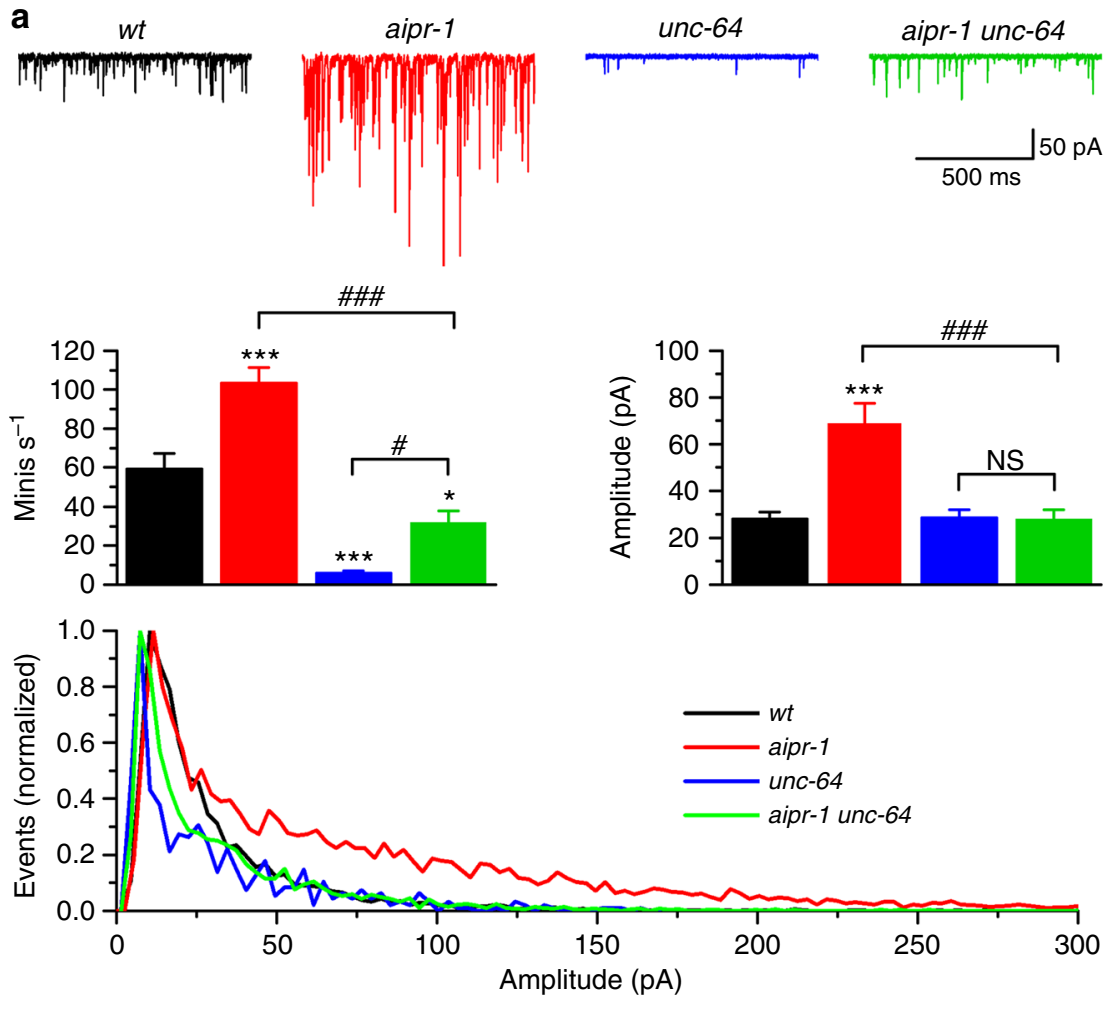

b
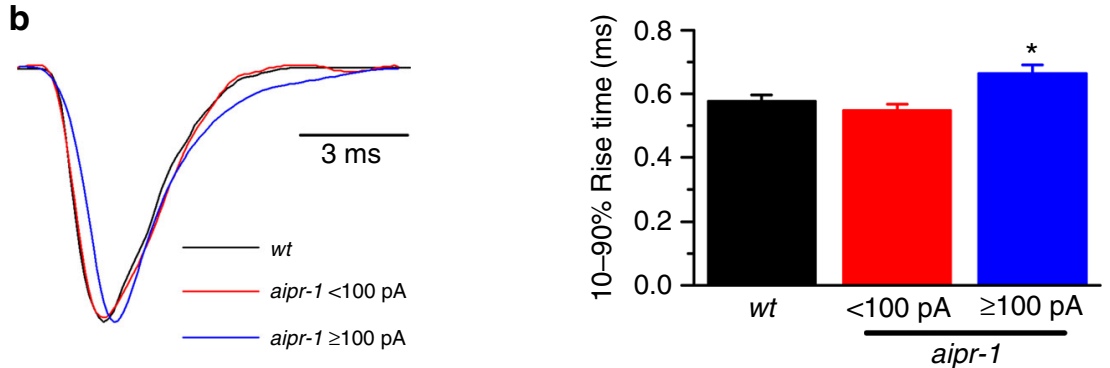

Fig. 6 AIPR-1 inhibits synchrony of spontaneous release. a Mutation of unc-64 (syntaxin) precluded the enhancing effect of aipr-1(zw86) on the amplitude of spontaneous miniature currents (minis). Frequency, mean amplitude, and amplitude distribution of minis are compared among the wild type ( $w t)$ ( $n=$ 9), aipr-1(zw86) (n=7), unc-64(e246) ( $n=9)$, and aipr-1(zw86) unc-64(e246) ( $n=8)$. It is noteworthy that the aipr-1 mutation increased large-amplitude events in the amplitude distribution graph and this effect was eliminated by a mutation in unc-64. b Comparison of $10-90 \%$ rise time of minis between wild type (wt) and aipr-1(zw86). The sample minis were normalized in amplitude and superimposed at an identical time scale. Data are shown as mean \pm SEM ${ }^{\star} p<0.05,{ }^{\star \star \star} p<0.001$ compared with wt; ${ }^{\#} p<0.05,{ }^{\# \# \#} p<0.001$, and NS ( $p>0.05$ ) compared between indicated groups (one-way ANOVA followed by Tukey's post-hoc test)

Previous studies have shown that activation of presynaptic ryanodine receptors leads to large minis by promoting synchronous multivesicular release $e^{6,7}$. If large minis are caused by multivesicular release, rather than increases in vesicle filling or postsynaptic responses, then decreasing release probability using mutations in the SNARE (soluble N-ethylmaleimide-sensitive factor attachment protein receptor) fusion machinery should restore mini amplitude. Syntaxin is encoded by the unc-64 gene in C. elegans ${ }^{44}$ and the hypomorphic unc-64(e246) mutant exhibits reduced mini frequency ${ }^{8,45}$. In aipr-1(zw86) unc-64(e246) double mutant, mini frequency is reduced and mini amplitudes are restored to wild-type levels (Fig. 6a), suggesting that large minis in the aipr-1 mutant are likely due to multivesicular release. Furthermore, we analyzed the rise times of minis, which are predicted to increase if large minis are due to multivesicular release $^{6,7}$. Our previous study shows that, in wild-type worms, all minis are mono-quantal events with a similar rise time regardless of the amplitude ${ }^{8}$. We divided minis of the aipr-1 mutant into two categories based on amplitudes $(<100 \mathrm{pA}$ and $\geq 100 \mathrm{pA})$, and compared the mean rise times with the wild type. We found that the rise time of large minis in the aipr-1 mutant was significantly longer than that of wild-type minis (Fig. 6b). Collectively, these observations suggest that the large-amplitude minis in the aipr-1 mutant are caused by synchronous multivesicular release as a result of elevated $\mathrm{Ca}^{2+}$ concentration at the presynaptic terminal, and that the function of AIPR-1 at the synapse is to reduce calcium bursts mediated by the ryanodine receptor.

To determine whether AIPR-1 might physically interact with RYR-1, we performed bimolecular fluorescence complementation (BiFC) assays ${ }^{46}$. The N-terminal portion of YFP (YFPa) was fused to the $\mathrm{C}$ terminus of the ryanodine receptor RYR-1 and the Cterminal portion of YFP (YFPc) was fused to the $\mathrm{C}$ terminus of AIPR-1 (Fig. 7a). We observed YFP fluorescence when the two 
a

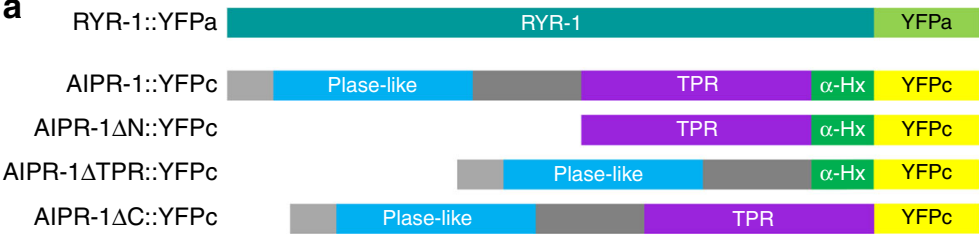

b
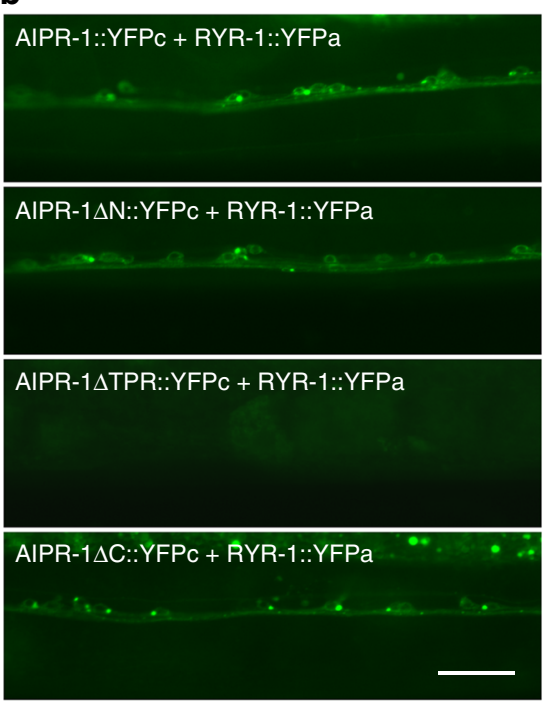

C

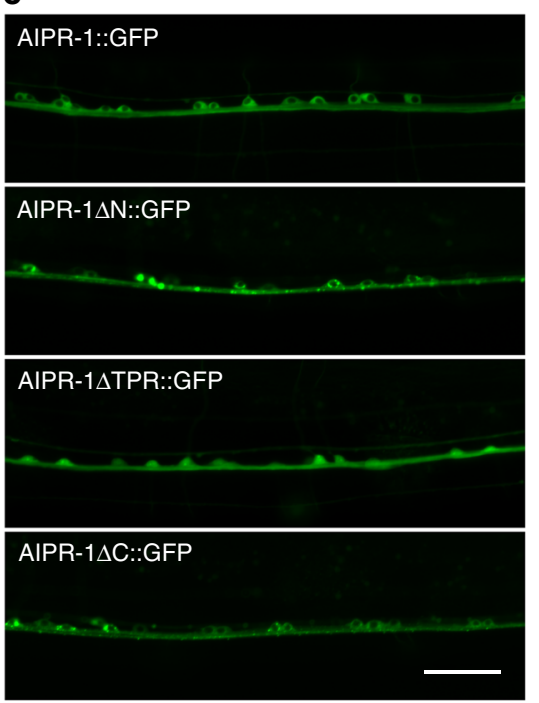

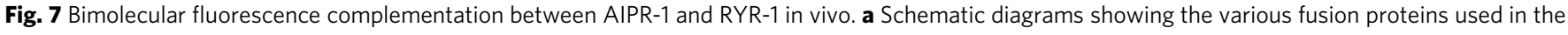
bimolecular fluorescence complementation assays. b Deletion of the TPR domain but not any other parts of AIPR-1 prevented AIPR-1::YFPc from reconstituting YFP fluorophore with RYR-1::YFPa in ventral cord motor neurons. c GFP-tagged AIPR-1 with the various deletions showed comparable expression levels in ventral cord motor neurons. Scale bars, $20 \mu \mathrm{m}$

fusion proteins were coexpressed in neurons (Fig. 7b). YFP fluorescence was also observed when either the prolyl isomerase domain or the C-terminal $\alpha-7$ helix was deleted (Fig. 7b). However, YFP fluorescence was not detected when the TPR domain was deleted (Fig. 7b). The lack of BiFC with the TPR deletion is unlikely due to a lack of protein expression, because this deletion did not appreciably alter the expression level of a GFP fusion protein (Fig. 7c). These results suggest that AIPR-1 is physically associated with RYR-1 and this association requires the TPR domain. This is consistent with an established role of TPR domains in mediating protein-protein interactions ${ }^{47}$ and with the loss-of-function phenotype of $z w 86$, which disrupts the last TPR motif (Fig. 1).

aipr-1 mutants accumulate vesicles independent of RYR-1. To determine whether changes in vesicle number or size are contributing factors to aipr-1(zw86) synaptic phenotypes, we performed ultrastructural analyses and quantified the number and size of synaptic vesicles at GABA and acetylcholine synapses. The aipr-1(zw86) mutant exhibits a nearly twofold increase in the number of synaptic vesicles (GABA neurons, Fig. 8a, b and Supplementary Fig. 10d'; ACh neurons, Supplementary Figs. 9 and 10a,d). Docked vesicle numbers also trend upward, although to a lesser degree (GABA neurons, Fig. 8c and Supplementary Fig. 10c'; ACh neurons, Supplementary Fig. 10b,c). Interestingly, ryr-1(lf) does not mask these effects (total vesicles: $w t 23.1 \pm 2.1$; aipr-1 $40.1 \pm 2.2 ; \quad$ ryr-1 $27.2 \pm 2.8 ; \quad$ aipr-1 $\quad$ ryr-1 $43.4 \pm 2.6$; docked vesicles: $w t 0.8 \pm 0.2$; aipr-1 $1.2 \pm 0.2$; ryr -10.8 \pm 0.2 ; aipr-1 ryr $-10.9 \pm 0.1$ ). These observations suggest that, besides regulating $\mathrm{Ca}^{2+}$ release by RYR-1, AIPR-1 has effects on vesicle biogenesis.
To determine whether these docked vesicles were primed, we stimulated release using a hypertonic sucrose solution (Fig. 8d). This $\mathrm{Ca}^{2+}$-independent response assesses the size of the readily releasable pool by driving exocytosis of docked vesicles ${ }^{48}$. The readily releasable pool is increased in aipr-1 using this assay as well, the sucrose response was increased $\sim 3$-fold in aipr-1(zw86) compared with the wild type (wt $40.5 \pm 8.5 \mathrm{pC}$; aipr-1 $148.6 \pm$ $16.6 \mathrm{pC}$ ). Similar to the increase in total vesicles, the increase in the readily releasable pool is not caused by dysregulation of the ryanodine receptor: the sucrose response was increased by more than twofold in the aipr-1 ryr-1 double mutant compared with ryr-1 (ryr-1 $42.9 \pm 8.9 \mathrm{pC}$; aipr-1 ryr-1 $97.3 \pm 16.8 \mathrm{pC})$. The enhanced sucrose response in aipr-1 mutant was completely abolished by a mutation of UNC-13/Munc-13, which has a pivotal role in synaptic vesicle priming ${ }^{49,50}$, suggesting that the increase in primed vesicles does not bypass the normal mechanisms for docking and priming.

\section{Discussion}

The striking increase in neurotransmitter release seen in the aipr1 mutant demonstrates that the AIPR-1/AIP protein is a major inhibitor of neurotransmission. Loss of AIPR-1/AIP results in increases in several aspects of neurotransmitter release, including evoked responses, spontaneous vesicle fusion, and current amplitude of spontaneous events. These effects can be attributed to disinhibition of the ryanodine receptor and the concomitant increase in calcium release from internal stores. In addition to the ryanodine receptor, AIP must act on at least one other target that limits synaptic vesicle number at neuromuscular junctions. Given that the mouse AIP can rescue the synaptic phenotypes of aipr-1 mutant, the regulation of ryanodine receptors by AIPR-1/AIP is likely conserved between worms and mammals. 

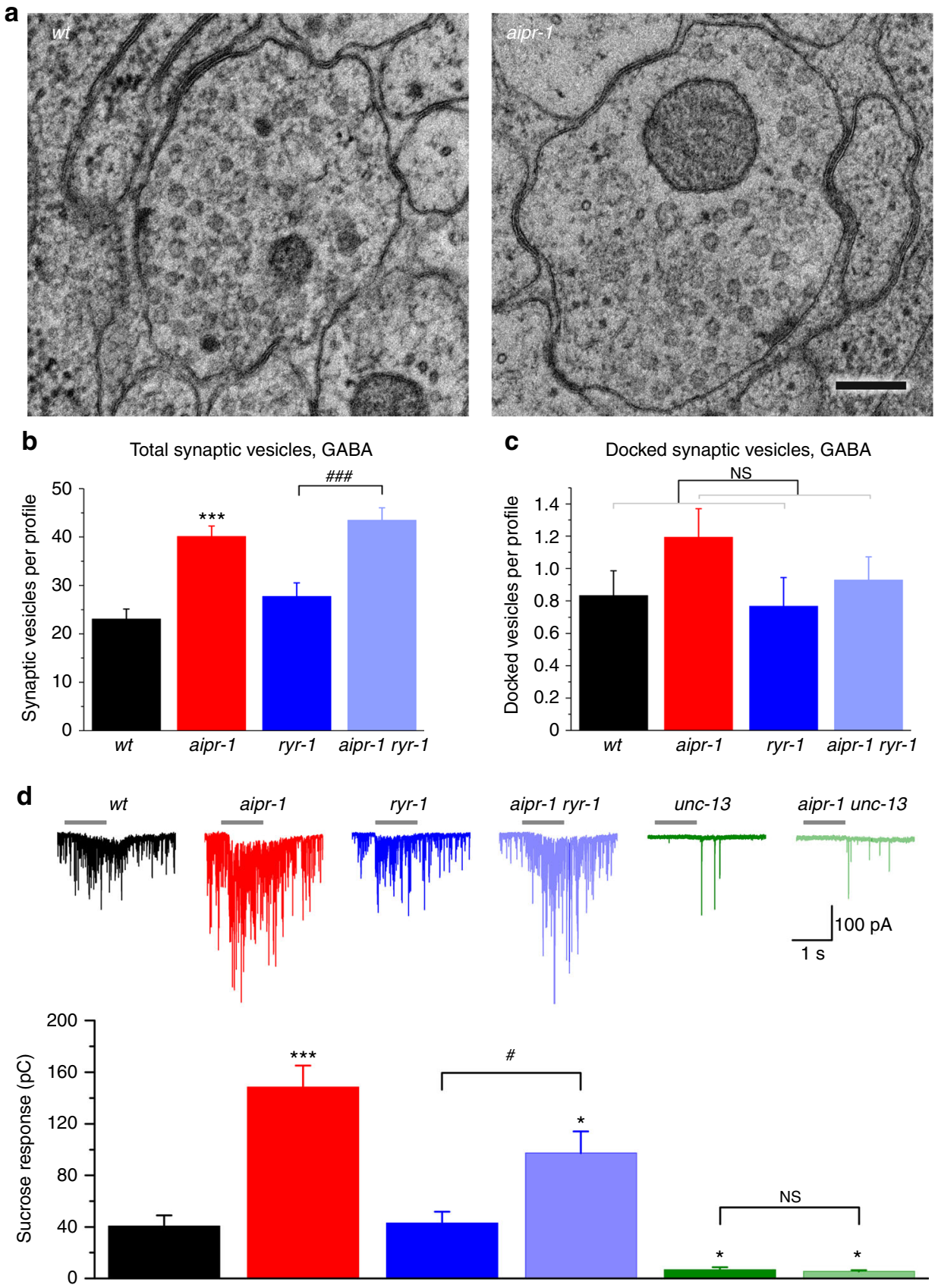

Fig. 8 Effects of AIPR-1 deficiency on synaptic vesicle number and the size of the readily releasable pool. a Sample micrographs of synapses from wild-type and aipr-1(zw86) animals. b, c airp-1(zw86) increases the numbers of synaptic vesicles $\mathbf{b}$ and docked vesicles $\mathbf{c}$. Increases were observed regardless of the ryr-1 genotype. Vesicle numbers compared at GABA synapses among wt $(n=66$ synaptic profiles), aipr-1(zw86) $(n=31), r y r-1(e 540)(n=30)$, and aipr-1 (zw86) ryr-1(e540) ( $n=56)$. d aipr-1(zw86) augmented sucrose-evoked postsynaptic currents at the neuromuscular junction regardless of the presence of ryr-1 mutation. The sample size was wt $(n=7)$, aipr-1(zw86) $(n=8)$, ryr-1(e540) $(n=7)$, aipr-1(zw86) ryr-1(e540) $(n=8)$, unc-13(s69) $(n=7)$, and aipr-1

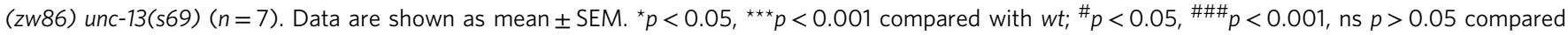
between indicated groups (one-way ANOVA followed by Tukey's post-hoc test or Welch's two-tailed $t$-test (c) generalized linear model, Poisson family: airpr-1(zw86) effect $p=0.077$, ryr-1(e540) effect: NS. Scale bar, $150 \mathrm{~nm}$

The increase in vesicles in aipr-1 mutants results in a substantial 3.2-fold increase in primed vesicles as assayed by hypertonic sucrose. Although the increase in docked vesicles (1.4fold) seems modest by comparison, this value represents docking in single profiles of neuromuscular junctions. Accurate counts of docked vesicles per synapse would require full reconstructions of motor neuron contacts along the muscle. Importantly, the increase in vesicles is not caused by increased calcium release from ryanodine receptors: mutation of RYR-1 does not reverse the increase in vesicles or the increase in the sucrose response observed in aipr-1 mutant. At this point it is unclear why vesicles accumulate in aipr-1 mutant. It is possible that vesicle biogenesis is increased or that vesicle turnover is slowed, or that homeostatic mechanisms of the synapses are disrupted.

The increases in neurotransmitter release, by contrast, can be fully attributed to dysregulation of the ryanodine receptor. The disinhibition of the ryanodine receptor in the aipr-1 knockdown worms can be directly observed as a doubling of calcium bursts at 
synapses. Knockdown of the ryanodine receptor completely eliminates the excessive calcium transients caused by knockdown of aipr-1, demonstrating that the calcium barrages arise from internal calcium stores. These calcium bursts are the probable cause of the twofold increase in spontaneous mini frequency observed in the aipr-1 mutant. It is likely to be that the ryanodine receptor is also hypersensitive to calcium, as electrical stimulation results in a 2.3-fold increase in evoked responses, suggesting that calcium influx through voltage-gated channels is eliciting a larger response from the ryanodine receptors. Finally, these calcium bursts are driving multiquantal release, as reflected in the 2.7 -fold increase in mini amplitudes.

\section{Table 1 List of worm strains}

Strain ID Genotype

Wild type N2 Bristol

CB246 unc-64(e246) III

CB540 ryr-1(e540) $\mathrm{V}$

CB55 unc-2(e55) $X$

BC168 unc-13(s69) I

EN208 unc-29(kr208::tagRFP) I

ZW029 slo-1(md1745) V

ZW083 zwls101[Pslo-1::slo-1::GFP (wp5)]

ZW320 zwls129[Pslo-1::slo-1(gf) (wp708); Pmyo-2::YFP (pPD132.102)]

ZW742 aipr-1(zw86); zwls101[Pslo-1::slo-1::GFP (wp5)]

ZW743 aipr-1(zw86)II; slo-1(md1745) V

ZW744 aipr-1(zw86) /I

ZW745 aipr-1(zw86) II; zwls129[Pslo-1::slo-1(gf) (wp708); Pmyo-2::YFP (wp214)]

ZW918

ZW929

ZW932

ZW936

ZW939

ZW940

ZW941 zwIs138[Prab-3::ryr-1::YFPa (wp1593 + wp1601), lin-15( + )]; zwEx205[Prab-3::aipr-1(41-192)::YFPc (wp1605), Pmyo-2::

aipr-1(zw86) II; zwEx195[Pmyo-3::aipr-1 (wp1466), Pmyo-2::YFP (pPD132.102)]

aipr-1(zw86) II; unc-64(e246) III

aipr-1(zw86) II; unc-2(e55) X

aipr-1(zw86) II; ryr-1(e540) V

aipr-1(zw86) II; zwls133[Punc-17::GFP (wp608)]

zwls138[Prab-3:::ryr-1::YFPa (wp1593 + wp1601), lin-15( + )]; zwEx204[Prab-3::aipr-1::YFPc (wp1500), Pmyo-2::

mStrawberry (wp1613)] mStrawberry (wp1613)]

ZW942 zwls138[Prab-3::ryr-1::YFPa (wp1593 + wp1601), lin-15( + )]; zwEx206[Prab-3::aipr-1(4193-308)::YFPc (wp1606), Pmyo2::mStrawberry (wp1613)]

ZW943 zwls138[Prab-3::ryr-1::YFPa (wp1593 + wp1601), lin-15( + )]; zwEx207[Prab-3::AIPR-1(4309-342)::YFPc (wp1607),

Pmyo-2::mStrawberry (wp1613)]

ZW944 aipr-1(zw86) II; oxls215[Punc-47::mRFP]

ZW948 zWEx208[Punc-4::aipr-1 senseRNA (wp1611), Punc-4::aipr-1 antisenseRNA (wp1612), Punc-4::mStrawberry (wp1400), Pmyo-2::mStrawberry (wp1613)]; zwEx196[Punc-1741::GCaMP6f (wp1600), lin-15( + )]

ZW949 ZwEx209[Punc-4::ryr-1 senseRNA (wp1609), Punc-4::ryr-1 antisenseRNA (wp1610), Punc-4::aipr-1 senseRNA (wp1611), Punc-4::aipr-1 antisenseRNA (wp1612), Punc-4::mStrawberry (wp1400), Pmyo-2::mStrawberry (wp1613)]; zwEx196[Punc1741::GCaMP6f (wp1600), lin15( + )]

ZW950 zwEx210[Pmyo-2::mStrawberry (wp1613), Punc-4::mStrawberry (wp1400)]; zwEx196[Punc-1741::GCaMP6f (wp1600), lin$15(+)]$

ZW951 ZwEx211[Punc-4::ryr-1 senseRNA (wp1609), Punc-4::ryr-1 antisenseRNA (wp1610), Punc-4::mStrawberry (wp1400), Pmyo2::mStrawberry (wp1613)]; zwEx196[Punc-1741::GCaMP6f (wp1600), lin-15( + )]

ZW1022 ZwEx232[Pryr-1b::GFP(wp1481), lin15( + )]; lin-15(n765)X

ZW1028 zwEx215[Prab-3::aipr-1::GFP (wp1465), lin-15( + )]; lin-15(n765) X

ZW1030 aipr-1(zw86) II; unc-13(s69) I

ZW1032 aipr-1(zw86) II; zwEx216[Prab-3::aipr-1 (wp1464), Pmyo-2::YFP (wp214)]

ZW1033 aipr-1(zw86) II; zWEx217[Prab-3::mAIP (wp1486), Pmyo-2::YFP (pPD132.102)]

ZW1098 zwEx233[Pryr-1a::GFP(wp149), lin15( + )]; $\operatorname{lin}-15(n 765) X$

ZW1102 aipr-1[zw88(GFP::aipr-1)] /I

ZW1103 aipr-1[zw89(aipr-1::GFP)] II

ZW1106 ZwEx235[Paipr-1::GFP(wp1747 + wp1750); lin-15( + )]; lin-15(n765) X

ZW1111 zwEx237[Pmyo-3::ryr-1 senseRNA (wp1745), Pmyo-3::ryr-1 antisenseRNA (wp1746), Pmyo-3::GFP(wp124)]; aipr-1(zw86) II

ZW1112 ZwEx238[Prab-3::mStrawberry::PISY-1(wp1387), Prab-3::GFP::ELKS-1(wp1753), lin-15( + )]; lin-15(n765) X

ZW1113

ZW1119

ZW1120

ZW1122

ZW1123

ZW1124

ZW1125

ZW1127

ZW1128

ZW1131 unc-29(kr208::tagRFP) l; aipr-1(zw86) II zwEx239[Paipr-1::GFP(wp1747 + wp1750), Punc-47::mStrawberry (wp1401), lin-15( + )]; lin-15(n765) X zwEx240[Paipr-1::GFP(wp1747 + wp1750), Punc-17::mRFP (wp865), lin-15( + )];lin-15(n765) X

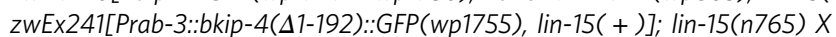
zwEx242[Prab-3::bkip-4(4193-308)::GFP(wp1756), lin-15( + )]; lin-15(n765) X zwEx243[Prab-3::bkip-4(A 309-342)::GFP(wp1757), lin-15( + )]; lin-15(n765) X zwEx244[Prab-3::aipr-1::GFP (wp1465, 2 ng/ $\mu$ l), Prab-3::TagRFP::elks-1 (wp1670, $5 \mathrm{ng} / \mu \mathrm{l}), \operatorname{lin}-15(+)$ ]; lin-15(n765) X zWEx245[Prab-3::GFP::ELKS-1 (wp1753), lin-15( + )]; lin-15(n765) X zwEx245[Prab-3::GFP:::ELKS-1 (wp1753), lin-15( + )]; aipr-1(zw86) II aipr-1(zw90)/ + II
Source

CGC

CGC

CGC

CGC

CGC

Jean-Louis Bessereau

Strains from CGC (Caenorhabditis Genetics Center) and other labs are indicated for their sources 
How might AIP regulate the activity of the ryanodine receptor? AIP belongs to the TPR family of co-chaperones that function with HSP90. In contrast to other heatshock proteins that function largely during protein synthesis or in degradation of unfolded proteins, HSP90 often forms stable interactions with intrinsically unstable proteins, such as many kinases and maintains them in a functional state ${ }^{51}$. HSP90 and its co-chaperone AHA1 protect the CFTR channel from thermal instability in the membrane ${ }^{52}$. In a similar manner, AIP may recruit HSP90 to the ryanodine receptor and stabilize the closed state of the ryanodine receptor. In the absence of AIP, the ryanodine receptor is unstable and spontaneously opens to generate calcium bursts from internal stores at the synapse.

Pituitary enlargement is likely an important factor in the hypersecretion of grown hormone in patients with AIP mutations ${ }^{53}$. Based on the results of this study, we speculate that increased calcium release from the ER due to disinhibition of the ryanodine receptor might also contribute to the acromegaly and gigantism phenotypes observed in these patients, although there are cases in which AIP mutations are not associated with hypersecretion of growth hormone ${ }^{24}$. Ryanodine receptors are expressed in the pituitary gland and mediate the release of growth hormone ${ }^{54,55}$ and results from goldfish pituitary cells show that pharmacological activation of ryanodine receptors can enhance growth hormone release ${ }^{56}$. Although we have uncovered a potential role for AIP in release at the C. elegans neuromuscular junction, further work in growth hormone-releasing cells is required to verify that AIP deficiencies can cause increased calcium bursts and growth hormone release.

The regulatory effects of AIPR-1 on RYR-1 are supported by our observations that they colocalize at synapses and are in physical contact. The BiFC assays indicate that AIPR-1 binds to RYR-1 through its TPR domain. Structural studies of AIP demonstrate that the TPR domain and the $\alpha-7$ helix motif are involved in HSP90 binding and in client protein binding ${ }^{21}$. By contrast, the calstabins lack a TPR domain and thus must interact with ryanodine receptors through their N-terminal region ${ }^{57,58}$.
The differences in overall structures and in the domains binding to ryanodine receptors suggest that AIPR-1/AIP and calstabins may regulate ryanodine receptor function through different mechanisms. However, it remains to be determined whether they can act cooperatively in inhibition of calcium release from internal stores under physiological conditions.

The present study establishes AIPR-1/AIP as a novel regulator of neurotransmitter release functioning through ryanodine receptors. Neurotransmitter is released by fusion of synaptic vesicles, whereas hormones are released by fusion of secretory granules. Nevertheless, the fusion machinery share many conserved molecules and features, such as the dependence on calcium and the use of the same set of SNARE proteins ${ }^{59}$. The demonstration that AIP is a negative regulator of calcium bursts at synapses opens the possibility that ryanodine receptor disinhibition in endocrine cells might affect secretion of growth hormone.

\section{Methods}

C. elegans culture and strains. All worms were raised on agar plates with a layer of OP50 Escherichia coli at $22^{\circ} \mathrm{C}$ inside an environmental chamber. Strains used in this study are listed in Table 1 .

Genetic Screen. A genetic screen was performed with an integrated transgenic strain (ZW320) expressing a hyperactive SLO-1 potassium channel, SLO-1(gf), which was created by mutating a single amino acid residue in the cytoplasmic gate of the S6 helix (E350Q) ${ }^{28}$. Synchronized L4-stage slo-1(gf) worms were treated with the chemical mutagen ethyl methanesulfonate $(50 \mathrm{mM})$ for $4 \mathrm{~h}$ at room temperature. The F2 progeny were screened for animals that moved better than the original slo-1 $(g f)$ animals. Forty mutants were isolated from screening over 100,000 haploid genomes. Complementation tests indicated that many of the mutants belong to genes previously implicated in SLO-1 function or subcellular localization $28,60,61$ $z w 86$ was among five mutants of genes not identified in previous SLO-1(gf)-based genetic screens. Single-nucleotide polymorphism-based genetic mapping ${ }^{62}$ of the $z w 86$ strain placed it in a region between $4,951,007$ and $11,827,835$ on chromosome II. A total of 15 genes within this interval have mutations in this strain based on whole-genome sequencing data. Through complementation tests and mutant rescuing experiments for the candidate genes, we identified $z w 86$ as an allele of C56C10.10. The behavioral phenotypes of $z w 86$ were rescued by a $5.2 \mathrm{~kb}$ genomic DNA fragment amplified from wild-type strain using primers $5^{\prime}$ -

\begin{tabular}{|c|c|}
\hline Gene & Sequence \\
\hline \multirow[t]{2}{*}{ 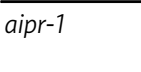 } & 5'-AATGGATCCGCCACCATGTCGGTCAGAGCAACTGT-3' (forward) \\
\hline & 5'-ATTACCGGTTATGGCTGAAACATTTTCGAAT-3' (reverse) \\
\hline \multirow[t]{2}{*}{ mAIP } & 5'- ATAAGATCTCCATGGCGGATCTCATCGCAAG-3' (forward) \\
\hline & 5'-TGTGCCGGCTCAGTGGGAAAAGATGCCC-3' (reverse) \\
\hline \multirow[t]{2}{*}{ Paipr-1 } & 5'- ATACTGCAGCGAGTGGCCGGATAATCTGA-3' (forward) \\
\hline & 5'- ATTACCGGTGCTCTGACCGACATTCTGAA-3' (reverse) \\
\hline \multirow[t]{2}{*}{ Pryr-1a } & 5'- GTGTTTTCCACGTCTGCGCCTTGCT-3' (forward) \\
\hline & 5'- ATATTGGCCATCGTTGTCGATCGTCGATCT-3' (reverse) \\
\hline \multirow[t]{2}{*}{ Pryr-1b } & 5'-TCTAAGATCTTGTCATCGTCAATGA-3' (forward) \\
\hline & 5'-ATTACCGGTACGCAGGAGAGGCAGACGAT-3' (reverse) \\
\hline \multirow[t]{2}{*}{ aipr-1 RNAi } & 5'-TTTGGTACCGTCGGTCAGAGCAACTGTGGT-3' (forward) \\
\hline & 5'-TATGCCGGCGCTGCGCACATGTGAGTTG-3' (reverse) \\
\hline \multirow[t]{2}{*}{ ryr-1 RNAi } & 5'-TTTGGTACCGGATGGGAGCAAGTGTTTCG-3' (forward) \\
\hline & 5'- TATGCCGGCTCCGTTGATTGGTTTCCTTCA-3' (reverse) \\
\hline \multirow[t]{2}{*}{ Pryr-1a } & 5'- GTGTTTTCCACGTCTGCGCCTTGCT-3' (forward) \\
\hline & 5'- ATATTGGCCATCGTTGTCGATCGTCGATCT-3' (reverse) \\
\hline \multirow[t]{2}{*}{ act-1 } & 5'- GCCCAATCCAAGAGAGGTATCC-3' (forward) \\
\hline & 5'- TGAGGAGGACTGGGTGCTCT-3' (reverse) \\
\hline \multirow[t]{2}{*}{ C56C10.9 } & 5'- TTCCCACAATCTCGACCCA-3' (forward) \\
\hline & 5'- CAATTCATCAACGGTTCCAGG-3' (reverse) \\
\hline \multirow[t]{2}{*}{$d n j-8$} & 5'- CGCCAATCCAAGAATTCGTT-3' (forward) \\
\hline & 5'- TGTCCATGCTCCTTCCAGC-3' (reverse) \\
\hline \multirow[t]{2}{*}{ C56C10.11 } & 5'- TCTTGCCAGTCAACCAGATCC-3' (forward) \\
\hline & 5'- GGAGGTCGAGAAAGGTGGGT-3' (reverse) \\
\hline \multirow[t]{2}{*}{ pisy-1 } & 5'- TAAGGTACCATGGAACCGACAGCCGCCGA-3' (forward) \\
\hline & 5'- ATAGCTAGCTCATTGGGCCTTCTGAGCAG-3' (reverse) \\
\hline
\end{tabular}


TTCCGGAGTCATGCTGTCC-3' (forward) and 5'- GTGCTGAGTATTCTGGGTGCAA $-3^{\prime}$ (reverse). The $z w 86$ mutant was outcrossed five times before analyses.

Rescue and knockout experiments. Wild-type aipr-1 cDNA (C56C10.10) was amplified from a Bristol N2 cDNA library and cloned into a worm expression vector containing either Prab-3 for neuron-specific rescue (prab-3::aipr-1, wp1464) or Pmyo-3 for muscle-specific rescue (Pmyo-3::aipr-1, wp1466). A mouse AIP cDNA (GenBank: BC075614.1) was amplified from a mouse brain cDNA library and cloned into the worm expression vector containing Prab-3 (Prab-3::mAIP, wp1486). The above plasmids were injected independently into aipr-1(zw86) mutant along with a Pmyo-2::YFP plasmid (pPD132.102), which served as a transformation marker. Primers for cloning aipr-1 and $m A I P$ cDNAs are listed in Table 2.

The CRISPR/Cas9 approach was used to create aipr-1 knockouts ${ }^{63}$. The guide RNA sequence (5'-GTGGTAAAACGGACAATCAG-3') targeting the first exon of aipr-1 was inserted into $\mathrm{P} u 6:$ :unc-119 sgRNA (Addgene plasmid 46169) to replace unc-119 sgRNA. The P $u 6$ ::aipr-1 sgRNA plasmid thus produced (wp1543) was coinjected with Peft-3::Cas9-SV40_NLS::tbb-2 (Addgene plasmid 46168) and Pmyo2::mStrawberry (wp1613) into wild-type worms. Transgenic animals were singled and a genomic DNA fragment of aipr-1 was amplified from the progeny for sequencing. Primers for amplifying and sequencing aipr-1 were $5^{\prime}$-CCATCTCG GATTCTACGCCA-3' (forward) and 5'-ACAAATCGAAGAGGTCGTGGAT-3' (reverse).

GFP tagging of AIPR-1 using Crispr/Cas9. We fused GFP to the N terminus and $\mathrm{C}$ terminus of AIPR-1, respectively, using the Crispr/Cas9 approach. The guide RNA sequences used for the $\mathrm{N}$ and $\mathrm{C}$ terminus insertions were $5^{\prime}$-ATGTCGGTCAGAGCAACTG-3' and 5'- GAACGGAGAGCCGAAAAGA-3', respectively. The plasmids and the transgenic worms were generated as described above. GFP insertion strains were first screened by PCR followed by sequencing to confirm correct insertions.

\section{Analysis of expression pattern and subcellular localization. The expression} pattern of aipr-1 was assessed using an in vivo homologous recombination approach. Specifically, a $0.5 \mathrm{~kb}$ genomic DNA fragment immediately upstream of aipr-1 initiation site was fused to GFP and the resultant plasmid (wp1747) was linearized and co-injected with a linearized fosmid (WRM065bd01), which contains the entire operon. Recombination was confirmed by PCR. To determine whether aipr-1 is expressed in acetylcholine or GABA neurons, the plasmids Punc17::mRFP (wp865) and Punc-47::mStrawberry (wp1401) were added to the above injection mixture, respectively. The expression pattern of $r y r-1$ was assessed by expressing GFP under the control of two alternative $r y r-1$ promoters (Pryr-1a and Pryr-1b). Primers for cloning Pryr-1a (2.5 kb) and Pryr-1b (4.8 kb) are listed in Table 2. Subcellular localization of AIPR-1 was determined by fusing GFP to its C terminus and expressing the fusion protein under the control of Prab-3 (Prab-3:: AIPR-1::GFP, wp1465). To determine whether AIPR-1 is enriched at presynaptic sites, a Prab-3::TagRFP::elks-1 plasmid (wp1670) was injected into the strain expressing Prab-3::aipr-1::GFP. ELKS-1 is localized at presynaptic sites in C. elegans neurons $^{64,65}$. Worms were immobilized with $10 \mathrm{mM}$ azide in M9 buffer. Images of transgenic worms were taken with a digital CMOS camera (Hamamatsu, C11440$22 \mathrm{CU}$ ) mounted on a Nikon TE2000-U inverted microscope (Nikon, Tokyo, Japan) equipped with enhanced GFP/fluorescein isothiocyanate (FITC) and mCherry/ Texas Red filter sets (49002 and 49008, Chroma Technology Corporation, Rockingham, VT, USA). Primers for cloning Paipr-1 are listed in Table 2.

Analyses of motor neuron gross morphology and synapse density. To determine whether motor neurons of the aipr-1(zw86) mutant were grossly abnormal in morphology, two integrated transgenic strains expressing GFP under the control of Punc-17 and mRFP under the control of Punc-47 were first established with wildtype worms. These two transgenes were then independently crossed into aipr-1 (zw86). The numbers of GFP-labeled acetylcholine and mRFP-labeled GABA ventral cord motor neurons, and the overall morphology of motor neuron processes (ventral cord, dorsal cord, and commissures) were compared between wild type and aipr-1(zw86). To determine whether the density of synapses is altered in aipr-1(zw86), whole-mount wild-type and aipr-1(zw86) were immunostained with a primary antibody for RIM ${ }^{64}$ and an Alexa Fluor 594-conjugated secondary antibody (Molecular Probes, Eugene, OR, USA). RIM-immunoreactive puncta in a randomly selected segment $(60 \mu \mathrm{m})$ of the dorsal cord of each worm was manually counted. Worms were visualized and imaged as described above. The exposure time was identical between wild type and aipr-1(zw86) in photographing.

BiFC assay. BiFC assays were performed by coexpressing RYR-1 and AIPR-1 tagged with the $\mathrm{N}$ - and C-terminal portions of YFP (YFPa and YFPc), respectively, in neurons. As the ryr-1 gene is rather large, the genomic DNA encompassing the neuronal isoforms of RYR-1 (K11C4.5b and K11C4.5d), which were determined through expressing promoter::GFP transcriptional fusions, were amplified as two separate fragments with $750 \mathrm{bp}$ overlap. The amplified upstream and downstream DNA fragments were independently cloned into two worm expression vectors containing Prab-3 and the DNA sequence encoding YFPa, respectively, to generate plasmids wp1593 and wp1601. In wp1601, the DNA sequence encoding RYR-1 Cterminal was fused in-frame to that encoding YFPa. These two plasmids were linearized and co-injected into lin-15(n765) to produce full-length ryr-1::YFPa fusion via in vivo homologous recombination. A lin-15 rescue plasmid was also coinjected to serve as a transformation marker. The ryr-1::YFPa transgene was integrated into the genome through $\gamma$-irradiation. A representative integrant was outcrossed four times and then injected independently with four different plasmids encoding Prab-3::AIPR-1::YFPc (wp1500), Prab-3::AIPR-1(41-192)::YFPc (wp1605), Prab-3::AIPR-1(4193-308)::YFPc (wp1606), and Prab-3::AIPR-1(4309342)::YFPc (wp1607). A Pmyo-2:: mStrawberry plasmid was coinjected to serve as a transformation marker. Epifluorescence of the transgenic worms was visualized and imaged as described above.

Recording of postsynaptic currents. All electrophysiological experiments were performed with adult hermaphrodites. Spontaneous and evoked postsynaptic currents were recorded from the C. elegans neuromuscular junction as described previously $y^{8,45}$. To measure the readily releasable pool of synaptic vesicles, PSCs in response to a hyperosmotic sucrose solution $(500 \mathrm{mM})$ were recorded from a body wall muscle cell. The sucrose solution was pressure-ejected $(1 \mathrm{~s}$ at $10 \mathrm{Psi})$ onto the ventral nerve cord near the recorded muscle cell through a glass pipette using a Picospritzer III Microinjection Dispense System (Parker Hannifin Precision Fluidics Division, Hollis, NH, USA). Two different extracellular solutions and two different pipette solutions were used, as specified in figure legends. Extracellular solution I contained (in $\mathrm{mM}$ ) $\mathrm{NaCl} 140, \mathrm{KCl} 5, \mathrm{CaCl}_{2} 5, \mathrm{MgCl}_{2}$ 5, dextrose 11, and HEPES 5 ( $\mathrm{pH}$ 7.2). Extracellular solution II differed from extracellular solution I in that $\mathrm{CaCl}_{2}$ was reduced to $0.5 \mathrm{mM}$, whereas $\mathrm{NaCl}$ was increased to $145 \mathrm{mM}$. Pipette solution I contained (in $\mathrm{mM}$ ) $\mathrm{KCl} 120, \mathrm{KOH} 20$, Tris $5, \mathrm{CaCl}_{2} 0.25, \mathrm{MgCl}_{2} 4$, sucrose 36, EGTA 5, and $\mathrm{Na}_{2} \mathrm{ATP} 4$ (pH 7.2). Pipette solution II differed from pipette solution I in that $113.2 \mathrm{mM} \mathrm{KCl}$ was substituted by Kgluconate. Except when described otherwise in figure legend, all recordings were made with extracellular solution I and pipette solution I at a holding voltage of $-60 \mathrm{mV}$.

$\mathbf{C a}^{2+}$ imaging and RNAi. A codon-optimized GCaMP6f was synthesized and placed under the control of Punc-1741, in which the proximal portion $(-2,429$ to $-83 \mathrm{bp}$ ) of $\mathrm{P} u n c-17$ was deleted so that its promoter activity is restricted almost exclusively to acetylcholine motor neurons in the ventral nerve cord. A-type motor neuron-specific gene knockdown was achieved by coexpressing two plasmids encoding sense and corresponding antisense RNA fragments of the targeted gene under the control of Punc-4. The primers used for amplifying an aipr-1 and ryr-1 cDNA fragments are listed in Table 2. The above cDNA fragments of aipr-1 and ryr-1 were inserted into the Punc-4 expression vector in both orientations to make plasmids encoding corresponding sense and antisense cDNA fragments. The plasmids for aipr-1 and ryr-1 knockdown were injected into the strain expressing Punc-1741::GCaMP6f (wp1600), both separately and in combination, to create three independent transgenic strains. Punc-4::mStrawberry (wp1400) and Pmyo-2:: mStrawberry (wp1613) were coinjected to label A-type motor neurons and serve as a transformation marker, respectively. Young adult worms were immobilized on Sylgard-coated coverglasses by applying a tiny drop of Vetbond Tissue Adhesive (3M Company, St Paul, MN, USA) on the dorsal anterior part of the worm and immersed in extracellular solution I. Spontaneous fluorescence changes in motor neurons of a ventral cord segment anterior to the vulva were imaged at 16 frames per second for 2 min using an electron-multiplying charge-coupled device (CCD) camera (iXonEM +885 , Andor Technology, Belfast, Northern Ireland), a FITC/ Texas Red filter set (59222, Chroma Technology Corporation), a light source (Lambda XL, Sutter Instrument, Novato, CA, USA), and NIS-Elements software (Nikon).

Thrashing assay. A single young adult hermaphrodite was transferred to an unseeded NGM plate and overspread with $\sim 60 \mu \mathrm{l}$ of M9 buffer. After a $1 \mathrm{~min}$ recovery period from the transfer, snapshots of the worm were taken at 15 frames per second for $1 \mathrm{~min}$ using a VGA FireWire camera (XCD-V60, Sony, Tokyo, Japan) mounted on a stereomicroscope (SMZ800, Nikon). The worm was automatically kept near the center of the view field through a motorized microscope stage (OptiScan ES111, Prior Scientific, Rockland, MA, USA). Both the camera and the motorized stage were controlled by an automated worm tracking system running in MATLAB (The MathWorks, Natick, MA, USA). Thrashing was counted manually from playing back the movies at 5 frames per second. A "thrash" was defined as one head bending toward one side of the body axis.

High-pressure freezing. Young adult worms were high-pressure frozen with a HPM100 (Leica Microsystems, Buffalo Grove, IL, USA). To mount the worms for freezing, a $3 \mathrm{~mm}$ support ring was loaded into a standard $6 \mathrm{~mm}$ middle plate. A 3 mm sapphire disc (Technotrade International, Manchester, NH, USA) was placed in the support ring, and on the disc was placed a copper $1 \times 2 \mathrm{~mm}$ transmission electron microscope (TEM) slot grid (Ted Pella, Redding, CA, USA), wetted with hexadecene oil. Using a paintbrush (number 00), $\sim 10$ worms were transferred to the slot, using the OP50 bacteria as a space filler. A $6 \mathrm{~mm}$ B-type aluminum specimen carrier (Ted Pella), flat side down, capped off the assembly. After high- 
pressure freezing, the sample (typically still associated with the grid and sapphire) was liberated from the other pieces under $-90^{\circ} \mathrm{C}$ anhydrous acetone in an automatic freeze substitution chamber (AFS2, Leica Microsystems). The specimen was then transferred to a cryovial containing $1 \%$ osmium tetroxide, $1 \%$ glutaraldehyde, and $1 \%$ water in acetone (Electron Microscopy Sciences, Hatfield, PA, USA), already in the AFS chamber.

Freeze substation and sample processing. Freeze substitution was performed with the following program: $-90^{\circ} \mathrm{C}$ for at least $5 \mathrm{~h},+5^{\circ} \mathrm{C} / \mathrm{h}$ to $-20^{\circ} \mathrm{C},-20^{\circ} \mathrm{C}$ for $14 \mathrm{~h},+10^{\circ} \mathrm{C} / \mathrm{h}$ to room temperature. During the program, the vials were agitated at least twice a day, promoting efficient diffusion of the fixatives into the tissues. Once at room temperature, the samples were removed from the AFS chamber and washed with anhydrous acetone six times over $90 \mathrm{~min}$, stained en bloc with $0.1 \%$ uranyl acetate (Polysciences, Warrington, PA, USA), and washed again with anhydrous acetone six times over $90 \mathrm{~min}$. Next, the worms were nutated while infiltrating with Epon/Araldite resin (Ted Pella) in a stepwise manner: $30 \% 3 \mathrm{~h}$, $70 \% 4 \mathrm{~h}, 90 \%$ overnight at $4{ }^{\circ} \mathrm{C}$, and finally three exchanges of $100 \%, 2 \mathrm{~h}$ each Worms were dissociated from the bacteria and distributed about the bottom of an embedding capsule cap (Ted Pella). Resin was cured for $48 \mathrm{~h}$ at $60^{\circ} \mathrm{C}$. Worms were mounted on base blocks and trimmed in cross-section to the anterior reflex of their gonad. Ultrathin $(33 \mathrm{~nm}$ ) serial sections were cut using an EM UC7 (Leica Microsystems) and collected on pioloform-coated slot grids. Post staining was performed with $2.5 \%$ uranyl acetate in $70 \%$ methanol for $4 \mathrm{~min}$.

Microscopy. The ventral nerve cord of the animal was imaged at $120 \mathrm{keV}$ on a JEOL JEM-1400 Plus transmission electron microscope equipped with an Orius CCD camera (Gatan, Pleasanton, CA, USA). Images were collected from typically $\sim 300$ serial sections from each worm ( $n=2$ worms per genotype). Imaging and analysis were performed blind to genotype.

Morphometry. Morphometry was performed with the aid of custom ImageJ (NIH, Bethesda, MD, USA) and MATLAB (MathWorks) scripts (Shigeki Watanabe, M. Wayne Davis, Edward J. Hujber, and Erik M. Jorgensen). Synapses were defined as profiles containing a dense projection plus one adjacent profile on either side. Synaptic vesicles were classified as docked when in direct contact with the plasma membrane.

Data analyses. Amplitudes of evoked currents were quantified using Clampfit (version 10, Molecular Devices, Sunnyvale, CA, USA), whereas the frequency and mean amplitude of spontaneous minis using MiniAnalysis (Synaptosoft, Decatur, GA, USA), as described previously ${ }^{8,45}$. The current integral over $1.5 \mathrm{~s}$ upon the application of the hyperosmotic sucrose solution was quantified using Clampfit for statistical comparison. Calcium transients of A-type acetylcholine motor neurons were identified based on mStrawberry marker expression and synchronous activities $^{66}$. Transients were quantified by plotting out $F / F_{0}$ of each A-type motor neuron in the imaging field (typically three or four neurons) over the recording period and measuring the frequency, amplitude, and duration of peaks. Calcium transient data of all the imaged neurons in each prep were averaged to represent one sample for statistical analyses. The detection threshold was set at 0.05 above the baseline, and at least $3 \mathrm{~s}$ in duration. When quantifying the number of calcium transients that merged together, a drop of $F / F_{0}$ amplitude by at least $25 \%$ was used as the criteria to indicate that subsequent $F / F_{0}$ belonged to another calcium transient. Data graphing and statistical analyses were performed with OriginPro 2015 (OriginLab Corporation, Northampton, MA, USA). Data are shown as mean \pm SE. Either analysis of variance with Tukey's post-hoc test, $t$-test, or a generalized linear model was used for statistical comparisons as specified in figure legends. $p<$ 0.05 is considered to be statistically significant. The sample size $(n)$ equals to the number of cells, worms, or synaptic profiles analyzed.

Data availability. The data that support the findings of this study, including morphometry scripts, are available from the corresponding author upon reasonable request.

Received: 28 February 2017 Accepted: 6 October 2017

Published online: 09 November 2017

\section{References}

1. de Juan-Sanz, J. et al. Axonal endoplasmic reticulum Ca2+ content controls release probability in CNS nerve terminals. Neuron 93, 867-881 e866 (2017)

2. $\mathrm{Wu}, \mathrm{Y}$. et al. Contacts between the endoplasmic reticulum and other membranes in neurons. Proc. Natl Acad. Sci. USA 114, E4859-E4867 (2017).

3. Berridge, M. J. Neuronal calcium signaling. Neuron 21, 13-26 (1998).
4. Tsukita, S. \& Ishikawa, H. Three-dimensional distribution of smooth endoplasmic reticulum in myelinated axons. J. Electron Microsc. (Tokyo) 25, 141-149 (1976).

5. Collin, T., Marty, A. \& Llano, I. Presynaptic calcium stores and synaptic transmission. Curr. Opin. Neurobiol. 15, 275-281 (2005).

6. Llano, I. et al. Presynaptic calcium stores underlie large-amplitude miniature IPSCs and spontaneous calcium transients. Nat. Neurosci. 3, 1256-1265 (2000)

7. Sharma, G. \& Vijayaraghavan, S. Modulation of presynaptic store calcium induces release of glutamate and postsynaptic firing. Neuron 38, 929-939 (2003).

8. Liu, Q. et al. Presynaptic ryanodine receptors are required for normal quantal size at the Caenorhabditis elegans neuromuscular junction. J. Neurosci. 25, 6745-6754 (2005).

9. Del Prete, D., Checler, F. \& Chami, M. Ryanodine receptors: physiological function and deregulation in Alzheimer disease. Mol. Neurodegener. 9, 21 (2014).

10. Stutzmann, G. E. et al. Enhanced ryanodine receptor recruitment contributes to $\mathrm{Ca} 2+$ disruptions in young, adult, and aged Alzheimer's disease mice. J. Neurosci. 26, 5180-5189 (2006).

11. Zalk, R., Lehnart, S. E. \& Marks, A. R. Modulation of the ryanodine receptor and intracellular calcium. Annu. Rev. Biochem. 76, 367-385 (2007).

12. Lanner, J. T., Georgiou, D. K., Joshi, A. D. \& Hamilton, S. L. Ryanodine receptors: structure, expression, molecular details, and function in calcium release. Cold Spring Harb. Perspect. Biol. 2, a003996 (2010).

13. Kang, C. B., Hong, Y., Dhe-Paganon, S. \& Yoon, H. S. FKBP family proteins: immunophilins with versatile biological functions. Neurosignals 16, 318-325 (2008).

14. Wehrens, X. H. et al. FKBP12.6 deficiency and defective calcium release channel (ryanodine receptor) function linked to exercise-induced sudden cardiac death. Cell 113, 829-840 (2003).

15. Liu, X. et al. Role of leaky neuronal ryanodine receptors in stress-induced cognitive dysfunction. Cell 150, 1055-1067 (2012).

16. Yuan, Q. et al. Calstabin 2: An important regulator for learning and memory in mice. Sci. Rep. 6, 21087 (2016).

17. Lehnart, S. E. et al. Leaky Ca2+release channel/ryanodine receptor 2 causes seizures and sudden cardiac death in mice. J. Clin. Invest. 118, 2230-2245 (2008).

18. Umanskaya, A., Andersson, D., Xie, W., Reiken, S. \& Marks, A. R. C. elegans as a model to study ryanodine receptor function in aging (ABSTRACT). Biophys. J. 106, 111a (2014).

19. Das, A. K., Cohen, P. W. \& Barford, D. The structure of the tetratricopeptide repeats of protein phosphatase 5: implications for TPR-mediated proteinprotein interactions. EMBO J. 17, 1192-1199 (1998).

20. Trivellin, G. \& Korbonits, M. AIP and its interacting partners. J. Endocrinol. 210, 137-155 (2011).

21. Morgan, R. M. et al. Structure of the TPR domain of AIP: lack of client protein interaction with the C-terminal alpha-7 helix of the TPR domain of AIP is sufficient for pituitary adenoma predisposition. PLoS ONE 7, e53339 (2012).

22. Vierimaa, O. et al. Pituitary adenoma predisposition caused by germline mutations in the AIP gene. Science 312, 1228-1230 (2006).

23. Raitila, A. et al. Mice with inactivation of aryl hydrocarbon receptor-interacting protein (Aip) display complete penetrance of pituitary adenomas with aberrant ARNT expression. Am. J. Pathol. 177, 1969-1976 (2010).

24. Chahal, H. S., Chapple, J. P., Frohman, L. A., Grossman, A. B. \& Korbonits, M. Clinical, genetic and molecular characterization of patients with familial isolated pituitary adenomas (FIPA). Trends Endocrinol. Metab. 21, 419-427 (2010).

25. Ozfirat, Z. \& Korbonits, M. AIP gene and familial isolated pituitary adenomas. Mol. Cell Endocrinol. 326, 71-79 (2010).

26. Rostomyan L, Beckers A. Screening for genetic causes of growth hormone hypersecretion. Growth Horm. IGF Res. 30-31, $52-57$ (2016).

27. Fukuda, T. et al. Augmented growth hormone secretion and Stat 3 phosphorylation in an aryl hydrocarbon receptor interacting protein (AIP)disrupted somatotroph cell line. PLoS ONE 11, e0164131 (2016).

28. Chen, B. et al. A novel auxiliary subunit critical to BK channel function in Caenorhabditis elegans. J. Neurosci. 30, 16651-16661 (2010).

29. Wang, S. J. \& Wang, Z. W. Track-A-Worm, an open-source system for quantitative assessment of $C$. elegans locomotory and bending behavior. PLoS ONE 8, e69653 (2013).

30. Chen, B. et al. $\alpha$-Catulin CTN-1 is required for BK channel subcellular localization in C. elegans body-wall muscle cells. EMBO J. 29, 3184-3195 (2010).

31. Chen, B., Liu, P., Zhan, H. \& Wang, Z. W. Dystrobrevin controls neurotransmitter release and muscle $\mathrm{Ca}^{2+}$ transients by localizing $\mathrm{BK}$ channels in Caenorhabditis elegans. J. Neurosci. 31, 17338-17347 (2011).

32. Lin, B. C. et al. Deletion of the aryl hydrocarbon receptor-associated protein 9 leads to cardiac malformation and embryonic lethality. J. Biol. Chem. 282, 35924-35932 (2007). 
33. Pinan-Lucarre, B. et al. C. elegans Punctin specifies cholinergic versus GABAergic identity of postsynaptic domains. Nature 511, 466-470 (2014)

34. Richmond, J. E. \& Jorgensen, E. M. One GABA and two acetylcholine receptors function at the C. elegans neuromuscular junction. Nat. Neurosci. 2, 791-797 (1999).

35. Shou, W. et al. Cardiac defects and altered ryanodine receptor function in mice lacking FKBP12. Nature 391, 489-492 (1998).

36. Marx, S. O. et al. PKA phosphorylation dissociates FKBP12.6 from the calcium release channel (ryanodine receptor): defective regulation in failing hearts. Cell 101, 365-376 (2000)

37. Wu, B., Yamaguchi, H., Lai, F. A. \& Shen, J. Presenilins regulate calcium homeostasis and presynaptic function via ryanodine receptors in hippocampal neurons. Proc. Natl Acad. Sci. USA 110, 15091-15096 (2013).

38. Sakube, Y., Ando, H. \& Kagawa, H. An abnormal ketamine response in mutants defective in the ryanodine receptor gene ryr-1 (unc-68) of Caenorhabditis elegans. J. Mol. Biol. 267, 849-864 (1997).

39. Schafer, W. R. \& Kenyon, C. J. A calcium-channel homologue required for adaptation to dopamine and serotonin in Caenorhabditis elegans. Nature 375, 73-78 (1995)

40. Brenner, S. The genetics of Caenorhabditis elegans. Genetics 77, 71-94 (1974).

41. Richmond, J. E., Weimer, R. M. \& Jorgensen, E. M. An open form of syntaxin bypasses the requirement for UNC-13 in vesicle priming. Nature 412, 338-341 (2001).

42. Saheki, Y. \& Bargmann, C. I. Presynaptic $\mathrm{Ca}_{\mathrm{V}} 2$ calcium channel traffic requires CALF-1 and the $\alpha_{2} \delta$ subunit UNC-36. Nat. Neurosci. 12, 1257-1265 (2009).

43. Chen, T. W. et al. Ultrasensitive fluorescent proteins for imaging neuronal activity. Nature 499, 295-300 (2013).

44. Saifee, O., Wei, L. \& Nonet, M. L. The Caenorhabditis elegans unc-64 locus encodes a syntaxin that interacts genetically with synaptobrevin. Mol. Biol. Cell 9, 1235-1252 (1998).

45. Wang, Z. W., Saifee, O., Nonet, M. L. \& Salkoff, L. SLO-1 potassium channels control quantal content of neurotransmitter release at the C. elegans neuromuscular junction. Neuron 32, 867-881 (2001).

46. Hu, C. D., Chinenov, Y. \& Kerppola, T. K. Visualization of interactions among bZIP and Rel family proteins in living cells using bimolecular fluorescence complementation. Mol. Cell 9, 789-798 (2002).

47. Zeytuni, N. \& Zarivach, R. Structural and functional discussion of the tetratrico-peptide repeat, a protein interaction module. Structure 20, 397-405 (2012).

48. Rosenmund, C. \& Stevens, C. F. Definition of the readily releasable pool of vesicles at hippocampal synapses. Neuron 16, 1197-1207 (1996).

49. Augustin, I., Rosenmund, C., Sudhof, T. C. \& Brose, N. Munc13-1 is essential for fusion competence of glutamatergic synaptic vesicles. Nature 400, 457-461 (1999).

50. Richmond, J. E., Davis, W. S. \& Jorgensen, E. M. UNC-13 is required for synaptic vesicle fusion in C. elegans. Nat. Neurosci. 2, 959-964 (1999).

51. Taipale, M. et al. Quantitative analysis of HSP90-client interactions reveals principles of substrate recognition. Cell 150, 987-1001 (2012).

52. Bagdany, M. et al. Chaperones rescue the energetic landscape of mutant CFTR at single molecule and in cell. Nat. Commun. 8, 398 (2017).

53. Daly, A. F. et al. Clinical characteristics and therapeutic responses in patients with germ-line AIP mutations and pituitary adenomas: an international collaborative study. J. Clin. Endocrinol. Metab. 95, E373-383 (2010).

54. Sawisky, G. R. \& Chang, J. P. Intracellular calcium involvement in pituitary adenylate cyclase-activating polypeptide stimulation of growth hormone and gonadotrophin secretion in goldfish pituitary cells. J. Neuroendocrinol. 17, 353-371 (2005)

55. Velazquez-Marrero, C. et al. mu-Opioid inhibition of Ca2+currents and secretion in isolated terminals of the neurohypophysis occurs via ryanodinesensitive Ca2+stores. J. Neurosci. 34, 3733-3742 (2014).

56. Johnson, J. D., Klausen, C., Habibi, H. R. \& Chang, J. P. Function-specific calcium stores selectively regulate growth hormone secretion, storage, and mRNA level. Am. J. Physiol. Endocrinol. Metab. 282, E810-819 (2002).

57. Lee, E. H. et al. N-terminal region of FKBP12 is essential for binding to the skeletal ryanodine receptor. J. Biol. Chem. 279, 26481-26488 (2004).
58. Samso, M., Shen, X. \& Allen, P. D. Structural characterization of the RyR1FKBP12 interaction. J. Mol. Biol. 356, 917-927 (2006).

59. Xu T, Xu P. in Molecular Mechanisms of Neurotransmitter Release (ed. Wang, Z.W.) 327-339 (Humana Press, 2008).

60. Sancar, F. et al. The dystrophin-associated protein complex maintains muscle excitability by regulating $\mathrm{Ca}(2+)$-dependent $\mathrm{K}(+)(\mathrm{BK})$ channel localization. $J$. Biol. Chem. 286, 33501-33510 (2011).

61. Kim, H. et al. The dystrophin complex controls bk channel localization and muscle activity in Caenorhabditis elegans. PLoS Genet. 5, e1000780 (2009).

62. Davis, M. W. et al. Rapid single nucleotide polymorphism mapping in $C$. elegans. BMC Genomics 6, 118 (2005).

63. Dickinson, D. J., Ward, J. D., Reiner, D. J. \& Goldstein, B. Engineering the Caenorhabditis elegans genome using Cas9-triggered homologous recombination. Nat. Methods 10, 1028-1034 (2013).

64. Deken, S. L. et al. Redundant localization mechanisms of RIM and ELKS in Caenorhabditis elegans. J. Neurosci. 25, 5975-5983 (2005).

65. Zheng, Q. et al. The vesicle protein SAM-4 regulates the processivity of synaptic vesicle transport. PLoS Genet. 10, e1004644 (2014).

66. Kawano, T. et al. An imbalancing act: gap junctions reduce the backward motor circuit activity to bias C. elegans for forward locomotion. Neuron 72, 572-586 (2011).

\section{Acknowledgments}

We thank the Caenorhabditis Genetics Center for mutant strains, Jean-Louis Bessereau for EN208 strain, and Fred Adler for advice on statistics. Supported by National Institute of Health grants R01-MH085927 (Z.-W.W.), R01-GM113004 (B.C.), and R01-NS034307 (E.M.J.). E. M. J. is an Investigator of the Howard Hughes Medical Institute (HHMI).

\section{Author contributions}

B.C. did molecular, cellular, and behavioral experiments. P.L. did electrophysiological experiments. E.J.H. did electron microscopy. Y.L. did mutant screen and mapping. B.C. and Z.-W.W. designed experiments. B.C., Z.-W.W. and E.M.J. wrote the manuscript.

\section{Additional information}

Supplementary Information accompanies this paper at doi:10.1038/s41467-017-01704-Z

Competing interests: The authors have no competing financial interests.

Reprints and permission information is available online at http://npg.nature.com/ reprintsandpermissions/

Publisher's note: Springer Nature remains neutral with regard to jurisdictional claims in published maps and institutional affiliations.

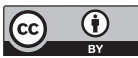

Open Access This article is licensed under a Creative Commons Attribution 4.0 International License, which permits use, sharing, adaptation, distribution and reproduction in any medium or format, as long as you give appropriate credit to the original author(s) and the source, provide a link to the Creative Commons license, and indicate if changes were made. The images or other third party material in this article are included in the article's Creative Commons license, unless indicated otherwise in a credit line to the material. If material is not included in the article's Creative Commons license and your intended use is not permitted by statutory regulation or exceeds the permitted use, you will need to obtain permission directly from the copyright holder. To view a copy of this license, visit http://creativecommons.org/ licenses/by/4.0/.

(c) The Author(s) 2017 